University of Wollongong

Research Online

Australian Institute for Innovative Materials -

Papers

Australian Institute for Innovative Materials

$1-1-2014$

Strain-responsive polyurethane/PEDOT:PSS elastomeric composite fibers with high electrical conductivity

Mohammad Ziabari Seyedin

University of Wollongong, smzs934@uowmail.edu.au

Joselito M. Razal

University of Wollongong, jrazal@uow.edu.au

Peter C. Innis

University of Wollongong, innis@uow.edu.au

Gordon G. Wallace

University of Wollongong, gwallace@uow.edu.au

Follow this and additional works at: https://ro.uow.edu.au/aiimpapers

Part of the Engineering Commons, and the Physical Sciences and Mathematics Commons

Research Online is the open access institutional repository for the University of Wollongong. For further information contact the UOW Library: research-pubs@uow.edu.au 


\title{
Strain-responsive polyurethane/PEDOT:PSS elastomeric composite fibers with high electrical conductivity
}

\author{
Abstract \\ It is a challenge to retain the high stretchability of an elastomer when used in polymer composites. \\ Likewise, the high conductivity of organic conductors is typically compromised when used as filler in \\ composite systems. Here, it is possible to achieve elastomeric fiber composites with high electrical \\ conductivity at relatively low loading of the conductor and, more importantly, to attain mechanical \\ properties that are useful in strain-sensing applications. The preparation of homogenous composite \\ formulations from polyurethane (PU) and poly(3,4-ethylenedioxythiophene):poly(styrenesulfonate) \\ (PEDOT:PSS) that are also processable by fiber wet-spinning techniques are systematically evaluated. \\ With increasing PEDOT:PSS loading in the fiber composites, the Young's modulus increases exponentially \\ and the yield stress increases linearly. A model describing the effects of the reversible and irreversible \\ deformations as a result of the re-arrangement of PEDOT:PSS filler networks within PU and how this \\ relates to the electromechanical properties of the fibers during the tensile and cyclic stretching is \\ presented.
}

\section{Keywords}

electrical, strain, responsive, fibers, polyurethane, high, conductivity, elastomeric, pss, pedot, composite

Disciplines

Engineering | Physical Sciences and Mathematics

\section{Publication Details}

Seyedin, M. Ziabari., Razal, J. M., Innis, P. C. \& Wallace, G. G. (2014). Strain-responsive polyurethane/ PEDOT:PSS elastomeric composite fibers with high electrical conductivity. Advanced Functional Materials, 24 (20), 2957-2966. 
DOI: 10.1002/adfm.((please insert DOI)

\title{
Strain-responsive polyurethane/PEDOT:PSS elastomeric composite fibers with high electrical conductivity
}

By Mohammad Ziabari Seyedin, Joselito M. Razal*, Peter C. Innis, and Gordon G. Wallace*

M.Z. Seyedin, Dr. J.M. Razal, A/Prof. P.C. Innis, Prof. G.G. Wallace

Intelligent Polymer Research Institute

ARC Centre of Excellence for Electromaterials Science

AIIM Facility, Innovation Campus

University of Wollongong

Wollongong NSW 2522 (Australia)

E-mail: jrazal@uow.edu.au,gwallace@uow.edu.au

Keywords: conducting elastomeric fiber, strain sensor, polyurethane, PEDOT:PSS, wetspinning

\begin{abstract}
It is a challenge to retain the high stretchability of an elastomer when used in polymer composites. Likewise, the high conductivity of organic conductors is typically compromised when used as filler in composite systems. Here, we demonstrate that it is possible to achieve elastomeric fiber composites with high electrical conductivity at relatively low loading of the conductor, and more importantly, attain mechanical properties that are useful in strainsensing applications. We have systematically evaluated how to prepare homogenous composite formulations from polyurethane (PU) and poly(3,4-
\end{abstract}


ethylenedioxythiophene):poly(styrenesulfonate) (PEDOT:PSS) that are also processable by fiber wet-spinning technique. We have found that with increasing PEDOT:PSS loading in the fiber composites, the Young's modulus increased exponentially and the yield stress increased linearly. A model describing the effects of the reversible and irreversible deformations as a result of the re-arrangement of PEDOT:PSS filler networks within PU and how this relates to the electromechanical properties of the fibers during the tensile and cyclic stretching is presented.

\section{Introduction}

Fibers with high electrical conductivities have been produced from a range of organic

conductors, such as polyaniline, ${ }^{[1-3]} \quad$ polypyrrole, ${ }^{[4-6]} \quad$ poly(3,4ethylenedioxythiophene):poly(styrenesulfonate) (PEDOT:PSS), ${ }^{[7,8]}$ carbon nanotubes, ${ }^{[9-12]}$ and recently from graphene and graphene oxide. ${ }^{[13-15]}$ Despite being highly conducting, these fibers have very low deformability, possess relatively little to no elasticity or elastic recovery and can typically be stretched by no more than $20 \%$ of their original lengths. On the other hand, fibers with very high deformations and elasticity, typically produced from elastomers, are electrically insulating. The combination of high stretchability and high conductivity is important in applications requiring strain sensing such as wearable bionics ${ }^{[16-21]}$ and for stretchable circuits, ${ }^{[22]}$ and electrochromic textiles. ${ }^{[23]}$ For strain sensing applications, where the change in electrical resistance is measured as a function of applied strain, it is highly desirable for this response to be measurable in a wide range of applied strain. In practical situations, the applied strains can be as small as less than a few percent or as large as tens to hundreds of percent. Some applications where small strains are important are damage detection, structural characterisation, and fatigue studies in materials, ${ }^{[24-26]}$ while applications such as body movement measurement, ${ }^{[16,20]}$ medical monitoring, ${ }^{[18,19,27]}$ and sports 
rehabilitation and injury prevention ${ }^{[21,28]}$ require large strains sensing. This work reports for the first time, the production of elastomeric composite fibers with high electrical conductivity that are capable of monitoring wide range of applied strains (up to $260 \%$ ) and can therefore be useful in applications such as strain gauge sensors in wearable bionics and stretchable electronics. The fiber wet-spinning approach that we have developed efficiently exploits the high stretchability of a medical grade elastomeric polyurethane (PU) and the high conductivity of PEDOT:PSS.

This work contributes to the relatively unexplored production of conductive and elastomeric composite fibers. The lack of progress in this field can be attributed to the limited development of elastomeric composite formulations that contain well-dispersed conducting fillers, which are also processable by fiber wet-spinning methods. In the limited cases where conductive fillers such as polyaniline, ${ }^{[29]}$ polythiophene $^{[30]}$ and carbon nanotubes ${ }^{[31]}$ were utilized in the wet-spinning of conducting fiber composites, the observed change in the electrical properties have only been marginal at large deformations. These reports also show that significant amount of filler loading is necessary to achieve percolation of conducting networks, which was found to be detrimental to the overall mechanical properties of the composite. The most common alternative approach to produce fibers with both elastic and conductive properties is by coating the elastomeric fiber (some reports use yarn or textile) with a thin layer of the conducting material. This approach has been used for conductor/elastomer combinations such as polypyrrole on Tactel/Lycra ${ }^{[32]}$ and nylon/Lycra ${ }^{[21]}$ fabrics and PEDOT:PSS on Spandex fabric ${ }^{[23]}$ to name a few, employing methods such as oxidative chemical polymerization, in situ chemical polymerization, chemical vapor deposition, and dip-coating. However, the conductive coatings produced often have poor adhesion and are less stretchy than the parent elastomer fiber resulting in poor overall electrical and mechanical performance. ${ }^{[21,23,32]}$ The surface and mechanical property mismatch 
between the conductive and the elastomeric components is avoided in the approach presented in this work. Here we demonstrate an efficient method to prepare homogeneous composite formulations of well-dispersed PEDOT:PSS and PU. These composite formulations are easily processed via wet-spinning method to produce fibers with mechanical properties and electrical conductivities that are significantly higher than previous reports on elastomeric composites. These fibers can respond to a wide dynamic range of large strains and offer great potential for applications in motion sensing, biomedical monitoring, and stretchable electronics.

\section{Results and discussions}

\subsection{Fiber spinning}

The quality of the PU/PEDOT:PSS composite formulations and the choice of coagulation bath influence the rate of fiber formation, and ultimately the fiber morphology and properties. In the first instance, we have identified the important wet-spinning conditions necessary to prepare composite fibers that retain the elastomeric and highly conducting properties inherent to PU and PEDOT:PSS, respectively.

\subsubsection{Spinning formulations}

The difference in dispersability of PU and PEDOT:PSS in various organic solvents is expected to influence the ability to prepare homogeneous PU/PEDOT:PSS composite formulations for fiber spinning purposes.

DMF, THF and DMSO were the candidate solvents that showed the best dispersability for both PU and PEDOT:PSS and were therefore used to compare the processability of the spinning formulations. The use of DMF and DMSO in making PEDOT:PSS formulations, resulted in dispersions that are stable for weeks with particle size lower than that of equivalent dispersion in water (see Figure S1 in Supporting Information). It was also noted that the 
particle size of PEDOT:PSS dispersions in DMSO increased with concentration (Figure S2), which suggests that high PEDOT:PSS concentrations may not be ideal for fiber spinning process even when DMSO is used. The equivalent dispersion in THF contained significantly larger particles with noticeable formation of large aggregates that precipitate within the first week.

The addition of PU to PEDOT:PSS dispersions resulted in homogeneous PU/PEDOT:PSS composite formulations that were stable for months after preparation. When the three PEDOT:PSS dispersions were cast into films, the highest conductivity was achieved from the DMSO dispersion (Figure S3), which suggests that spinning formulations in DMSO would yield composite fibers with the highest conductivities. DMSO was then used for all subsequent experiments.

\subsubsection{Fiber spinning conditions}

The spinnability of PU/PEDOT:PSS composite formulations was evaluated using coagulation baths (non-solvents) that have worked well for fiber spinning of pure PU and pure PEDOT:PSS dispersions. ${ }^{[8]}$ When isopropanol was used as the non-solvent, coagulation was slow and beads formed at the tip of the spinneret that caused the fibers to break. The coagulation rate was improved dramatically when water used as coagulation bath but the fibers produced were electrically insulating. For the latter case, it was observed that phase segregation occurred, preventing the formation of a continuous conductive network. The use of isopropanol/water mixtures resulted in continuous fiber spinning and prevented phase segregation. The highest fiber conductivity achieved when $80 / 20 \mathrm{v} / \mathrm{v}$ isopropanol/water mixture was used.

Pure PU dispersions were found to be spinnable over a broad range of concentrations (from 30 to $250 \mathrm{mg} \mathrm{mL}^{-1}$ ) with concentrations above $50 \mathrm{mg} \mathrm{mL}^{-1}$ resulting in uniform fibers and reproducible spinnability. Therefore, in order to prepare composite formulations with high 
PEDOT:PSS loadings (up to 16.7 wt. \%) that have consistent spinnability, the $50 \mathrm{mg} \mathrm{mL}^{-1} \mathrm{PU}$ concentration was employed (Table 1). For comparison purposes, we have also prepared spinning formulations from lower PU concentrations to achieve PEDOT:PSS loading as high as 33.3 wt. \% (Table 1). A typical wet-spun PU/PEDOT:PSS fiber is shown in Figure S4. Composite formulations with PU concentration of $30 \mathrm{mg} \mathrm{mL}^{-1}$ and more, equivalent to PEDOT:PSS content of 25.0 wt. \% and lower, remained spinnable and resulted in continuous fiber formation. However, the formulation with PU concentration of $20 \mathrm{mg} \mathrm{mL}^{-1}$ loaded with 33.3 wt. \% PEDOT:PSS (No. 10) did not produce continuous fibers.

\subsubsection{Fiber morphology}

The representative SEM images of fibers shown in Figure 1 illustrate the change in fiber morphology, from an irregularly shaped cross-section of PU fiber to a more circularly shaped composite fibers. In fiber wet-spinning, fibers tend to have non-circular cross-sections when the diffusion of the coagulating solvent into the injected spinning solution is higher than the extraction rate of the solvent of spinning solution (into the bath), a process normally referred to as the mass transfer rate difference. ${ }^{[33]}$ Under the experimental conditions employed in this work, it was observed that the morphological changes occurred due to the differences in the coagulation rates arising from the various spinning formulation compositions. This means that the addition of PEDOT:PSS in spinning solution influences the mass transfer rate differences, whereby the phase transformation of the viscous spinning solution to a solid fiber occur at various rates for different PEDOT:PSS concentrations. In the case of wet-spinning of pure PU solutions, the difference in mass transfer rate favored the formation of a fiber with a solid skin and a core that remained relatively viscous. The PU fiber can collapse into an irregular shape when the remaining solvent is extracted. When PEDOT:PSS was added to the spinning formulation, the slower coagulation rate resulted in the thinner skin formation. Therefore, the fiber cross-section remained circular when all solvent was extracted. It was observed that the 
cross-section of composite fibers became circular when the PEDOT:PSS loading reached 13 wt. \% (Figure 1). Careful examination of the composite fibers (Figure 1i $-\mathbf{j}$ ) showed no aggregates, suggesting that the PEDOT:PSS is homogeneously dispersed within the PU host. These results are important because they reflect the quality of the homogeneity of the spinning formulations, which is expected to be directly translated to the fiber properties.

\subsection{Tensile properties}

\subsubsection{Stress-strain behavior}

Shown in Figure 2 are representative stress-strain curves of pure PU and PU/PEDOT:PSS composite fibers. It can be seen that both fibers display similar tensile behaviors reminiscent of an elastomeric material. The initial stiff behavior is followed by a compliant deformation that extends to high strains and finally, by a stress amplification before fracture. It can also be observed that compared to the pure PU fiber, the composite fibers have higher Young's modulus and yield stress but have lower tensile strength, elongation at break and toughness.

The observed tensile behavior of our wet-spun PU fiber is similar to the literature reports. This tensile behavior can be explained by the unique polymer chain composition of PU comprising soft and hard segments whereby the soft segments provide high elongation while hard segments provide stiffness. ${ }^{[34-38]}$ Therefore, the initial stiff response stems solely from the rigid hard segment domains. The compliant behavior observed at mid-range strains is attributed to the combination of soft segment extension, fibrillar hard segment orientation in the direction of the strain, and lamellar hard segment domain rotation perpendicular to the strain direction. ${ }^{[35,37,39]}$ The strain-hardening observed at high elongation is due mainly to strain-induced crystallization in soft segments. ${ }^{[35,37]}$ For our composite fibers, we believe that the addition of PEDOT:PSS to PU increases the rigid component content thereby increasing the stiffness and yield stress. However, this addition also influences the structural heterogeneity of the resulting composite and affects the strain-induced crystallization process 
in the soft segments at high elongation. These structural changes could explain the observed decrease in both elongation at break and tensile strength in the composite fibers. It is also possible that the interactions between PU and PEDOT:PSS result in significant morphological changes under load and cause early failure of the composite.

\subsubsection{Effect of PEDOT:PSS loading}

Figure 3 shows the mechanical properties of PU/PEDOT:PSS composite fibers with various PEDOT:PSS loadings. It can be observed that the Young's modulus of the composite fibers increased exponentially with PEDOT:PSS loading from 7.2 MPa (for pure PU) to 247 MPa (for the composite fiber with 25 wt. \% PEDOT:PSS content, Figure 3a). This increase is equivalent to more than a 30 -fold reinforcement in modulus. It is important to note that the high PEDOT:PSS loading (above 16.7 wt. \%) was achieved by decreasing the PU concentration in the spinning solution (see secondary x-axis). It was found that the modulus data fits very well with Mooney’s equation ${ }^{[40]}$ (Equation 1). This model has been employed for elucidating the reinforcement of fillers with relatively low stiffness and rigidity in a soft

matrix. ${ }^{[41-44]}$ According to this model, the Young's modulus of a composite $\left(E_{c}\right)$ at a volume fraction of filler $(\phi)$ can be described by:

$$
E_{c}=E_{p} \exp \left(\frac{k_{E} \phi}{1-s \phi}\right)
$$

where $E_{p}$ is the Young's modulus of the polymer, $k_{E}$ is the Einstein coefficient defined by the aspect ratio of the filler, and $s$ is the crowding factor defined by the ratio of occupied volume/true volume of the filler and varies between 1.35 and 1.91. A crowding factor closer to 1.91 means that the particle size of the filler is small and the fillers are homogeneously dispersed within the matrix. ${ }^{[41]}$ By fitting the data in Figure 3a with the Equation 1 ( $E_{p}$ of 7.19 MPa as for the pure PU fiber), we have found $s$ to be 1.85 suggesting that the small sizes 
of PEDOT:PSS particles in the spinning formulations are retained in the fiber composites and they also remained uniformly dispersed within the PU matrix after the fiber spinning process. These results are corroborated by our SEM observations and PEDOT:PSS particle size measurements. We have also calculated $k_{E}$ to be 7.61. For well-dispersed systems, $k_{E}$ has been reported to be 2.5 for spherical fillers ${ }^{[40,41]}$ and higher for fillers with higher aspect ratios, e.g. 40 in clay nanocomposites. ${ }^{[43]}$ Our $k_{E}$ suggests that the PEDOT:PSS are non-spherical and have an aspect ratio of $6.35 .^{[45]}$

The yield stress was obtained at $5 \%$ strain for each fiber. It was found that the yield stress increased linearly with PEDOT:PSS loading, achieving an approximately 20-fold increase for PEDOT:PSS content of 25 wt. \%. The yield stress for this composite fiber is $~ 9.1 \mathrm{MPa}$ compared to $~ 0.5 \mathrm{MPa}$ for pure PU fiber, Figure 3b.

Unlike Young's modulus and yield stress, the composite fibers' toughness, tensile strength, and elongation at break decreased rapidly with PEDOT:PSS loading above 9.1 wt. \% (Figure 3c-4e). Below this loading, the toughness, tensile strength and elongation at break did not change significantly. Also, contrasting the positive effect in Young's modulus and yield stress when the PU concentration is decreased in the spinning formulation to attain the highest PEDOT:PSS loading, the toughness, tensile strength and elongation at break decreased further.

These results (increase in Young's modulus and yield stress and decrease in tensile strength, elongation at break and toughness with increasing PEDOT:PSS loading) are similar to the behavior of other reinforced elastomeric composites, such as silica ${ }^{[46]}$, carbon nanotube, ${ }^{[47]}$ and graphene ${ }^{[48]}$ reinforced PU. This observed reinforcement in modulus and yield stress may be attributed to hydrodynamic effect ${ }^{[45,46]}$ with PEDOT:PSS being the more rigid component, as well as the physical and mechanical interactions ${ }^{[49,50]}$ between PEDOT:PSS and PU. These interactions, however, are not sufficient to withstand high loads to result in stress 
reinforcement at high elongations. Furthermore, at high elongations, it is likely that the extension, orientation and crystallization of the soft segments in the PU network is disrupted by the addition of PEDOT:PSS filler particles resulting earlier breakage of the fiber and lower tensile strength.

\subsection{Electromechanical properties}

\subsubsection{Elastic recovery behavior}

Figure 4a compares the elastic recovery behavior of pure PU and PU/PEDOT:PSS composite fibers. For both fibers, the loading and unloading paths of the stress-strain curves were different for each cycle suggesting hysteresis. The stress-strain curve of both fibers became more compliant during reloading indicative of stress softening behavior. This behavior is best illustrated by a lower stress in the second loading (reloading) curve than the first. The addition of PEDOT:PSS increased the magnitude of stress softening (stress reduced more in reloading).

It can also be seen that the origin of each of the reloading curves is shifted towards a strain higher than the origin of the preceding loading cycle, indicative of permanent set. The difference between the applied strain (i.e. the end point of the loading curve) and the permanent set for each loading-unloading cycle was used as a measure of elastic recovery. For each applied strain, it was found that the elastic recovery of the composite fibers decreased with PEDOT:PSS loading (Figure 4b). It can also be observed that the elastic recovery decreased sharply with PEDOT:PSS loading (from 95 \% to 65 \%) when the applied strain was low (at $50 \%$ strain). This relationship is depicted by the steepness of the best-fit line for each data set. It is important to note that the elastic recovery behavior of the composite fibers at various applied strains was different for each PEDOT:PSS loading (Figure S5). For pure PU and when the PEDOT:PSS loading in the composite fibers was less than 4.8 wt. \%, the 
elastic recovery decreased with increasing applied strain. At and above this loading, the elastic recovery initially increased with applied strain before eventually falling.

The above results can be explained as follows: In pure PU fiber, the loss of fibrillar and lamellar orientation, ${ }^{[35]}$ the irreversible deformations during loading, ${ }^{[35,51,52]}$ and the slippage of polymer chains in hard segment domains ${ }^{[53]}$ could account for the observed hysteresis and permanent set. The partial reconstruction of hard segment domains through the formation of new cross-links between polymer chains ${ }^{[37,54]}$ could explain the stress softening.

The observed behavior of PU/PEDOT:PSS composite fibers could be due to the weak interactions between PU and PEDOT:PSS, which suggest that the PEDOT:PSS polymer chains slip or break and do not recover or reform, respectively, during the unloading cycle. These effects become more pronounced when PEDOT:PSS loading is high because more PEDOT:PSS networks can fail under strain. Under high strain conditions, failure of PU hard segment domains accounts for majority of the decrease in elastic recovery because PEDOT:PSS networks fail at relatively low strains (depicted by the differences in slope in

\section{Figure 4b).}

\subsubsection{Elastic recovery behavior during cyclic stretching}

Fibers undergo cyclic stretching in strain sensing applications. We captured the cyclic behavior of the fibers by repeatedly stretching and relaxing the fiber at each applied strain for ten cycles with a $30 \mathrm{sec}$ hold period after each loading and unloading step (Figure 5). At each applied strain, Young's modulus decreased after each cycle with the degree of reduction being significantly higher between the first and the second cycle. Hysteresis and stress softening also decreased as the number of cycles increased, as most hysteresis and stress softening occurred in the first cycle. Larger hysteresis and stress softening were observed at higher applied strains suggesting their dependency on strain history. Furthermore, the unloading paths remained identical for all cycles in each applied strain. It was also observed that 
permanent set occurred mostly in the first cycle (i.e. the changes in the following cycles were insignificant). These observations are in agreement with the previous reports on cyclic behavior of polyurethane ${ }^{[55]}$ as well as filled elastomers. ${ }^{[56]}$

\subsubsection{Electrical conductivity}

The effect of PEDOT:PSS loading on the electrical conductivity of the composite fibers is shown in Figure 6. It was observed that the onset of conductivity occurred at $\sim 2.9$ wt. $\%$ PEDOT:PSS loading, achieving a conductivity of $\sim 0.07 \mathrm{~S} \mathrm{~cm}^{-1}$. Above this loading, the fiber conductivity increased monotonically with PEDOT:PSS loading and the highest conductivity of $\sim 25 \mathrm{~S} \mathrm{~cm}^{-1}$ was achieved at 25 wt. \% PEDOT:PSS.

Our experimental data fits well $\left(\mathrm{R}^{2}=0.998\right)$ with percolation theory described by Equation 2 , which shows that above the percolation threshold $\left(\phi_{c}\right)$, the conductivity $(\sigma)$ increases significantly with PEDOT:PSS filler content. This relationship suggests that there is a minimum amount of conducting filler needed to create conducting paths in an insulating matrix. At the filler loading below the percolation threshold, no conducting paths can be established because the average distance between filler particles is large and therefore charge carrier transport cannot occur. By increasing the filler loading, the average distance between filler particles decreases and below a certain threshold, charge carrier transport can occur via electrical field assisted tunneling or hopping between neighboring fillers. ${ }^{[57,58]}$ A further increase in filler loading brings particles in contact and creates conducting paths, enhancing the conductivity.

$$
\sigma=\sigma_{0}\left(\phi-\phi_{c}\right)^{t}
$$

We assumed that the densities of PU and PEDOT:PSS ${ }^{[59]}$ are both $1.2 \mathrm{~g} \mathrm{~cm}^{-3}$ to estimate the volume fraction $(\phi)$ for each PEDOT:PSS loading. Using Equation 2, a percolation threshold 
of 2.4 wt. \% PEDOT:PSS (equivalent to $\phi_{c}=0.024$ ) was derived from the fit which is in good agreement with the experimentally measured loading for onset of conductivity (2.9 wt. \%). From this equation, we also estimated the geometry of the conducting network defined by the critical exponent $t^{[57]}$ to be 1.27 . This $t$ value is very close to the theoretical value reported for two dimensional networks (1.33). ${ }^{[57]}$ Note that high $t$ values mean greater tunneling barriers between fillers and therefore, lower maximum composite conductivities. ${ }^{[60]}$

In most polymer composites, the conductive filler particles can become coated with a layer of polymer or can form heterogeneous aggregates. These situations often diminish the efficiency of charge transfer between the nearby conductive particles thereby resulting in a composite material with low electrical conductivities. A measure of the intrinsic conductivity of the filler in a percolating network can be estimated by the proportionality coefficient $\left(\sigma_{0}\right)$ in Equation 2, which was found to be $166 \mathrm{~S} \mathrm{~cm}^{-1}$ in our case. This value is very close to the conductivity of pristine PEDOT:PSS film, which suggests that the PEDOT:PSS is homogeneously dispersed in the PU matrix and are in good contact inside the PU host allowing for the full potential of PEDOT:PSS as the conductive filler to be attained.

We also note that the $\sigma_{0}$ for other conductive composites are significantly lower than in this work. For example, it has been reported that the $\sigma_{0}$ for polyvinyl alcohol (PVA)/carbon nanodisks composite is $0.40 \mathrm{~S} \mathrm{~cm}^{-1},^{[61]} 0.21 \mathrm{~S} \mathrm{~cm}^{-1}$ for PVA/multi-wall carbon nanotubes system, ${ }^{[61]} 0.79 \mathrm{~S} \mathrm{~cm}^{-1}$ for vinyl ester/carbon fibers ${ }^{[62]}$ and $0.33 \mathrm{~S} \mathrm{~cm}^{-1}$ for SIBS/P3HT fibers. ${ }^{[30]}$ To the best of authors' knowledge, the value of $\sigma_{0}\left(166.0 \mathrm{~S} \mathrm{~cm}^{-1}\right)$ obtained in this study is the highest value for elastomeric conducting composites and among the highest value reported in the literature with only polystyrene/graphene composite showing a higher value $\left(831.8 \mathrm{~S} \mathrm{~cm}^{-1}\right){ }^{[63]}$ 


\subsubsection{Strain sensing behavior}

It is clear from the above results that it is possible to retain the elastomeric behavior (inherent to PU) in a PU/PEDOT:PSS composite fiber, and at the same time, enhance its stiffness, yield stress and electrical conductivity (inherent to PEDOT:PSS). For example, the composite fiber with 13.0 wt. \% PEDOT:PSS loading is electrically conductive and has tensile properties and elastic recovery comparable to pure PU fiber. Owing to these reasonably balanced mechanical and electrical properties, this particular fiber was evaluated for strain sensing application and for elucidating the mechanism of its electromechanical behavior.

During the electromechanical cyclic test, it was observed that the resistance of the fiber was proportional with applied strain (Figure 7). The electrical resistance increased with applied strain of up to $260 \%$ after which the fiber became insulating (inset of Figure 7). It is also apparent from these data that the resistance follows a similar pattern in each cycle but shifts towards higher resistances compared to the preceding cycle.

The longitudinal sections of the PU/PEDOT:PSS fiber during cyclic deformations are schematically represented in Figure 8. These figures depict the state of the PEDOT:PSS networks in the elastomeric PU matrix, summarized as follows:

1. Unbreakable networks that do not fail under strain (•)

2. Reversible networks that if broken, recreate conductive networks when strain is released $(\bullet)$

3. Irreversible networks that if broken, do not recreate conductive networks even when strain is released $(0)$

4. Isolated networks that are unable to form conductive networks on their own (०)

5. Debonded networks with the potential to alter the conductivity (๑). ${ }^{[64]}$

It is envisaged that the interruption, deformation or re-arrangement of the above conductive filler networks during the strain and release periods as a result of their slippage, debonding 
and reversibility will determine the resistance response of the composite fiber. These instances are discussed in detail below.

Shown in Figure 9 is a profile of the change in resistance of the composite fiber in the first two cycles of applied strain. Each stretching zone is denoted by a number in this figure. Before stretching (Figure 9, zone 1), the fiber showed a resistance of $5.7 \mathrm{k} \Omega$. In this state, unbreakable $(\bullet)$, reversible $(\bullet)$, and irreversible $(\bullet)$ conductive networks are continuous (see conduction paths in Figure 8a). As a result, the fiber showed its minimum resistance that remained constant during the unstretched period. As the fiber is stretched in the first cycle (Figure 9, zone 2), the resistance increased due to the breakage of reversible ( $\bullet$ ) and irreversible ( $\bullet$ ) networks. The breakage of these two types of networks is illustrated by two boxes in Figure 8b (compare with the corresponding boxes in Figure 8a). During the hold period at strained state (Figure 9, zone 3), the stress distribution in the fiber can cause the PEDOT:PSS fillers to slip and re-arrange while the PU polymer chains relax. This can cause for the reversible and irreversible networks to break further. When the applied strain is released (Figure 9, zone 4), reversible (๑) networks can come in contact again (compare the boxes in Figure 8d with a) and establish conduction paths and result in a drop in resistance. In zone 5 of Figure 9, when the fiber is held at zero stress for $30 \mathrm{sec}$ during which creep occurs and strain is reduced, the resistance decreases similar to the zone 4 . Note that the resistance at the end of the first cycle (after unloading) was observed to be higher than that of the unstretched fiber. This increase may be due to the irreversible deformation of irreversible ( ○) networks (see boxes in Figure 8d and compare with Figure 8 a), and therefore reduction of conduction paths.

When the fiber is re-stretched in the second cycle (Figure 9, zone 6), reversible (๑) networks rupture again and more irreversible (๑) networks break down (similar to the first cycle). However, deformations take place more easily as the previously damaged networks may 
require less energy to break. This could be explained by the higher slope of the resistance curve in the re-stretching step. However, as the fiber is stretched, its cross-sectional area decreases bringing some debonded $(\odot)$ networks in contact and allowing interactions between other types of networks (compare the boxes in Figure 8c with the ones in Figure 8b). As a result, more conduction paths are created accounting for the observed decrease in resistance in the middle of this zone. Similar phenomenon occurs in Zone 7(the hold period at strained state in the second cycle) as in zone 3. In zone 8 (Figure 9), where the fiber is relaxed, the conducting paths are restored due to the reconnection of reversible $(\bullet)$ networks (same with Zone 4). At the same time, cross-section area of the fiber increases resulting in breakdown of some conductive networks that had been created from debonded (๑) networks and interactions of all networks (compare boxes in Figure 8c and b). This results in an increase in resistance, observed in the middle of this zone. In zone 9 (Figure 9), similar to the relaxation zone of the first cycle, resistance drops further with strain. The higher resistance at the end of the second cycle than in the first cycle could be due to the breakdown of more reversible networks. Some of the unbreakable $(\bullet)$ networks in the first cycle may also deform and change to other types in the second cycle, thereby failing in the subsequent cycles. It is also possible that some reversible networks become irreversible in the second cycle.

\section{Conclusions}

This work has demonstrated the production of conducting elastomeric composite fibers with wide strain sensing capabilities that may be suitable for applications in wearable bionics and stretchable electronics such as body movement measurement and biomedical monitoring. These composite fibers were easily prepared by ensuring that the spinning formulations used in fiber wet-spinning process are homogenous dispersions of the composite mixtures of PU and PEDOT:PSS and optimization of the solvent/non-solvent system. Using the optimized fiber spinning conditions, PU/PEDOT:PSS fibers with PEDOT:PSS content of up to $25.0 \mathrm{wt}$. 
$\%$ were fabricated. PU/PEDOT:PSS fibers exhibited high electrical conductivity and high Young's modulus, yield stress, and elastic recovery. Full potential of PEDOT:PSS as conducting filler in a composite system was achieved through maximum PEDOT:PSS particle contacts in PU. PU/PEDOT:PSS fibers responded to a wide range of cyclic stretching demonstrating merits for applications requiring strain sensing. The interruption, deformation and/or re-arrangement of PEDOT:PSS filler networks within the PU matrix are believed to be responsible for the observed changes in the electromechanical behavior of the fibers.

\section{Experimental}

\subsection{Materials}

PEDOT:PSS pellets (Agfa Orgacon ${ }^{\mathrm{TM}}$ Dry, Batch No. A06 0000BY) and polycarbonatebased biocompatible thermoplastic polyurethane (PU, AdvanSource Biomaterials Chronoflex ${ }^{\circledR} \mathrm{C}$ 80A) were used as received. N,N-dimethylformamide (DMF) purchased from Sigma-Aldrich and tetrahydrofuran (THF) and dimethylsulfoxide (DMSO) bought from Ajax Finechem, were used without further purifications.

\section{2. $\quad$ Spinning solution preparation and fiber spinning}

PU was dissolved in DMSO, DMF and THF (5 mL). PEDOT:PSS dispersions were prepared by homogenization (Labtek IKA T25) at $15000 \mathrm{rpm}$ for $30 \mathrm{~min}$ (water and DMSO) and homogenization and bath sonication (Branson B5500R-DTH) for 48 hrs (DMF and THF). PEDOT:PSS dispersion in DMSO was mixed with the PU solution at either a 1:1 or 2:1 v/v ratio (PEDOT:PSS/PU) to produce the desired PU/PEDOT:PSS formulation. The resulting formulation was stirred for $1 \mathrm{hr}$ prior to fiber spinning. Fiber spinning was carried out using the previously described wet-spinning setup for PEDOT:PSS fibers ${ }^{[8]}$ and SIBS/P3HT fibers. ${ }^{[30]}$ Briefly, the spinning solution was injected into the coagulation bath containing the non-solvents (i.e. water, isopropanol, or isopropanol/water mixture). A 23 gauge needle with 
blunt tip was used as a spinneret. The spinning solution flow rate was maintained at $5 \mathrm{~mL} \mathrm{~h}^{-1}$. Fibers were collected on a spool immediately after spinning.

\subsection{Characterization}

Particle size distributions in PEDOT:PSS dispersions were measured by dynamic light scattering technique with the aid of a Zetasizer (Malvern Instruments Nano-ZS) using a quartz cuvette. A minimum of 10 measurements were made for each sample. Fiber morphology was characterized using a field emission scanning electron microscope (JEOL JSM-7500FA). Samples were broken in liquid nitrogen and then sputter coated with gold (EDWARDS Auto 306). Fiber diameter was taken as the average of 10 points along the fiber length to be tested and was measured using an optical microscope (Leica DM6000 M). Fiber conductivity was measured using an in-house linear four-point probe cell with $230 \mu \mathrm{m}$ probe spacing. A galvanostat (Princeton Applied Research 363) was used to apply current between the outer probes and a digital multimeter (Agilent 34401A) was used to measure the voltage between the two inner probes. The mechanical and electromechanical properties of the fibers were evaluated using a tensile testing instrument (Shimadzu EZ-L) equipped with a $2 \mathrm{~N}$ load cell. Samples were prepared by attaching the fibers to paper frames (10 $\mathrm{mm}$ in aperture). Samples were then mounted on the sample holders ( $1 \mathrm{~N}$ clamps) and paper was cut once the frame was secured. For tensile tests, the strain rate (crosshead speed) was set to $10 \mathrm{~mm} \mathrm{~min}^{-1}$ (100\% $\min ^{-1}$ ) and fibers were stretched until failure. For the elastic recovery tests, fibers were first stretched to $50 \%$ (at a strain rate of $10 \mathrm{~mm} \mathrm{~min}^{-1}$ ) and were then relaxed with the same strain rate to zero stress. Fibers were subsequently stretched to $100 \%$, $200 \%$, $300 \%$, and $400 \%$, releasing to zero stress at the end of each applied strain. These tests were performed to measure the elastic recovery of the fibers. As a result of permanent set (unrecoverable strain due to plastic deformation), fibers do not reach their initial lengths after the strain is removed and consequently there is always a difference between the initial and the recovered strain. 
This difference was used to calculate the elastic recovery of the fibers at each applied strain. Cyclic tests were performed by first applying a $50 \%$ strain and then releasing to allow the fiber to return to the initial length (zero stress). This was repeated for 10 cycles with 30 sec relaxation time between each loading and unloading step. These tests were also extended to $100 \%$ and $200 \%$ strain. The strain sensing behavior of a selected PU/PEDOT:PSS fiber was investigated by monitoring the change in resistance using a digital multimeter (Agilent 34410A) when a strain is applied. This test was carried out in conjunction with the mechanical tests (tensile and cyclic) and the resistance data were captured every 0.05 sec during the tests using an A/D computer interface. The sample preparation was similar to the mechanical properties tests. Copper tape was placed at both ends to allow for resistance measurements.

\section{Supporting Information}

Supporting Information is available from the Wiley Online Library or from the authors.

\section{Acknowledgements}

This work is funded by the Australian Research Council (ARC) Centre of Excellence program and the University of Wollongong (UOW). JMR acknowledges funding from UOW URC grants and VC Fellowship. The authors thank the materials node of Australian National Fabrication Facility (ANFF) and acknowledge use of the facilities and the assistance of Mr. Tony Romeo at the UOW Electron Microscopy Centre.

Received: ((will be filled in by the editorial staff))

Revised: ((will be filled in by the editorial staff)) Published online: ((will be filled in by the editorial staff)) 


\section{References}

[1] A. Andreatta, Y. Caod, J. C. Chiang, A. J. Heeger, P. Smith, Synth. Met. 1988, 26, 383.

[2] S. J. Pomfret, P. N. Adams, N. P. Comfort, A. P. Monkman, Polymer 2000, 41, 2265.

[3] V. Mottaghitalab, B. Xi, G. M. Spinks, G. G. Wallace, Synth. Met. 2006, 156, 796.

[4] J. Foroughi, G. M. Spinks, G. G. Wallace, P. G. Whitten, Synth. Met. 2008, 158, 104.

[5] J. Foroughi, G. M. Spinks, G. G. Wallace, Synth. Met. 2009, 159, 1837.

[6] J. Foroughi, G. M. Spinks, G. G. Wallace, J. Mater. Chem. 2011.

[7] H. Okuzaki, Y. Harashina, H. Yan, Eur. Polym. J. 2009, 45, 256.

[8] R. Jalili, J. M. Razal, P. C. Innis, G. G. Wallace, Adv. Funct. Mater. 2011, 21, 3363.

[9] B. Vigolo, A. Pénicaud, C. Coulon, C. Sauder, R. Pailler, C. Journet, P. Bernier, P. Poulin, Science 2000, 290, 1331.

[10] A. B. Dalton, S. Collins, E. Muñoz, J. M. Razal, V. H. Ebron, J. P. Ferraris, J. N. Coleman, B. G. Kim, R. H. Baughman, Nature 2003, 423, 703.

[11] E. Munoz, A. B. Dalton, S. Collins, M. Kozlov, J. Razal, J. N. Coleman, B. G. Kim, V. H. Ebron, M. Selvidge, J. P. Ferraris, R. H. Baughman, Adv. Eng. Mater. 2004, 6, 801.

[12] A. B. Dalton, S. Collins, J. Razal, E. Munoz, V. H. Ebron, B. G. Kim, J. N. Coleman, J. P. Ferraris, R. H. Baughman, J. Mater. Chem. 2004, 14, 1.

[13] Z. Xu, C. Gao, Nat. Commun. 2011, 2, 571.

[14] X. Hu, Z. Xu, C. Gao, Sci. Rep. 2012, 2, 767.

[15] Z. Xu, H. Sun, X. Zhao, C. Gao, Adv. Mater. 2013, 25, 188.

[16] T. Yamada, Y. Hayamizu, Y. Yamamoto, Y. Yomogida, A. Izadi-Najafabadi, D. N. Futaba, K. Hata, Nat. Nanotechnol. 2011, 6, 296.

[17] R. Paradiso, G. Loriga, N. Taccini, IEEE Trans. Inf. Technol. Biomed. 2005, 9, 337.

[18] F. Carpi, D. De Rossi, Inf. Technol. Biomed. IEEE Trans. 2005, 9, 295.

[19] A. Lymberis, S. Olsson, Telemed. J. e-Health 2003, 9, 379. 
[20] A. Tognetti, F. Lorussi, R. Bartalesi, S. Quaglini, M. Tesconi, G. Zupone, D. De Rossi, J. Neuroeng. Rehabil. 2005, 2, 8.

[21] J. Wu, D. Zhou, C. O. Too, G. G. Wallace, Synth. Met. 2005, 155, 698.

[22] M. Park, J. Im, M. Shin, Y. Min, J. Park, H. Cho, S. Park, M.-B. Shim, S. Jeon, D.-Y. Chung, J. Bae, J. Park, U. Jeong, K. Kim, Nat. Nanotechnol. 2012, 7, 803.

[23] Y. Ding, M. A. Invernale, G. A. Sotzing, ACS Appl. Mater. Interfaces 2010, 2, 1588.

[24] I. Kang, M. J. Schulz, J. H. Kim, V. Shanov, D. Shi, Smart Mater. Struct. 2006, 15, 737.

[25] H. Zhao, Y. Zhang, P. D. Bradford, Q. Zhou, Q. Jia, F.-G. Yuan, Y. Zhu, Nanotechnology 2010, 21, 305502.

[26] X. Li, R. Zhang, W. Yu, K. Wang, J. Wei, D. Wu, A. Cao, Z. Li, Y. Cheng, Q. Zheng, R. S. Ruoff, H. Zhu, Sci. Rep. 2012, 2, 870.

[27] H. D. Kubo, B. C. Hill, Phys. Med. Biol. 1996, 41, 83.

[28] H. M. Mortensen, O. Skov, P. E. Jensen, J. Bone Joint Surg. Am. 1999, 81, 983.

[29] P. Sukitpaneenit, T. Thanpitcha, A. Sirivat, C. Weder, R. Rujiravanit, J. Appl. Polym. Sci. 2007, 106, 4038.

[30] A. J. Granero, P. Wagner, K. Wagner, J. M. Razal, G. G. Wallace, M. in het Panhuis, Adv. Funct. Mater. 2011, 21, 955.

[31] A. J. Granero, J. M. Razal, G. G. Wallace, M. in het Panhuis, In 2010 International Conference on Nanoscience and Nanotechnology; IEEE: Sydney, 2010; pp. 80-83.

[32] Y. Li, X. Y. Cheng, M. Y. Leung, J. Tsang, X. M. Tao, M. C. W. Yuen, Synth. Met. 2005, 155, 89.

[33] S. P. Mishra, A Textbook of Fibre Science and Technology; New Age International: New Delhi, 2000.

[34] B. Meissner, M. Špírková, Macromol. Symp. 2002, 181, 289.

[35] E. M. Christenson, J. M. Anderson, A. Hiltner, E. Baer, Polymer 2005, 46, 11744. 
[36] P. R. Laity, J. E. Taylor, S. S. Wong, P. Khunkamchoo, M. Cable, G. T. Andrews, A. F. Johnson, R. E. Cameron, Macromol. Mater. Eng. 2006, 291, 301.

[37] F. Yeh, B. S. Hsiao, B. B. Sauer, S. Michel, H. W. Siesler, Macromolecules 2003, 36, 1940.

[38] Z. S. Petrović, J. Ferguson, Prog. Polym. Sci. 1991, 16, 695.

[39] B. . Fu, B. . Hsiao, S. Pagola, P. Stephens, H. White, Polymer 2001, 42, 599.

[40] M. Mooney, J. Colloid Sci. 1951, 6, 162.

[41] T. B. Lewis, L. E. Nielsen, J. Appl. Polym. Sci. 1970, 14, 1449.

[42] S. Ahmed, F. R. Jones, J. Mater. Sci. 1990, 25, 4933.

[43] Y. Rao, J. M. Pochan, Macromolecules 2007, 40, 290.

[44] S.-Y. Fu, X.-Q. Feng, B. Lauke, Y.-W. Mai, Compos. Part B Eng. 2008, 39, 933.

[45] J. G. Brodnyan, J. Rheol. 1959, 3, 61.

[46] R. Nunes, J. Fonseca, M. Pereira, Polym. Test. 2000, 19, 93.

[47] F. M. Blighe, W. J. Blau, J. N. Coleman, Nanotechnology 2008, 19, 415709.

[48] U. Khan, P. May, A. O’Neill, J. N. Coleman, Carbon 2010, 48, 4035.

[49] A. Voet, J. Polym. Sci. Macromol. Rev. 1980, 15, 327.

[50] J. Fröhlich, W. Niedermeier, H.-D. Luginsland, Compos. Part A Appl. Sci. Manuf. 2005, 36, 449.

[51] D. Puett, J. Polym. Sci. Part A-2 Polym. Phys. 1967, 5, 839.

[52] G. Estes, R. Seymour, S. Cooper, Macromolecules 1971, 4, 452.

[53] E. M. Dannenberg, Trans. Inst. Rubber 1966, 42, 26.

[54] L. Bokobza, Macromol. Mater. Eng. 2004, 289, 607.

[55] H. J. Qi, M. C. Boyce, Mech. Mater. 2005, 37, 817.

[56] K. Yamaguchi, J. J. C. Busfield, A. G. Thomas, J. Polym. Sci. Part B Polym. Phys. 2003, 41, 2079. 
[57] D. Stauffer, A. Aharony, Introduction to Percolation Theory; 2nd ed.; CRC Press: London, 1994.

[58] R. Strumpler, J. Glatz-Reichenbach, J. Electroceramics 1999, 3, 329.

[59] R. Jalili, J. M. Razal, G. G. Wallace, J. Mater. Chem. 2012, 22, 25174.

[60] W. Bauhofer, J. Z. Kovacs, Compos. Sci. Technol. 2009, 69, 1486.

[61] Y. R. Hernandez, A. Gryson, F. M. Blighe, M. Cadek, V. Nicolosi, W. J. Blau, Y. K. Gun’ko, J. N. Coleman, Scr. Mater. 2008, 58, 69.

[62] J. Xu, J. P. Donohoe, C. U. Pittman, Compos. Part A Appl. Sci. Manuf. 2004, 35, 693.

[63] S. Stankovich, D. a Dikin, G. H. B. Dommett, K. M. Kohlhaas, E. J. Zimney, E. a Stach, R. D. Piner, S. T. Nguyen, R. S. Ruoff, Nature 2006, 442, 282.

[64] V. Jha, Carbon Black Filler Reinforcement of Elastomers, PhD Thesis: University of London, 2008. 
Table 1. PU/EDOT:PSS spinning formulations at different PEDOT:PSS contents

\begin{tabular}{cccc}
\hline Sample & PU & PEDOT:PSS & PEDOT:PSS Content \\
No. & {$\left[\mathrm{mg} \mathrm{mL}^{-1}\right]$} & {$\left[\mathrm{mg} \mathrm{mL}^{-1}\right]$} & {$[$ wt. $\%]$} \\
\hline 1 & 50 & 0.0 & 0.0 \\
3 & 50 & 1.0 & 2.0 \\
3 & 50 & 1.5 & 2.9 \\
4 & 50 & 2.5 & 4.8 \\
5 & 50 & 5.0 & 9.1 \\
6 & 50 & 7.5 & 13.0 \\
7 & 50 & 10.0 & 16.7 \\
8 & 40 & 10.0 & 20.0 \\
9 & 30 & 10.0 & 25.0 \\
10 & 20 & 10.0 & 33.3 \\
\hline
\end{tabular}




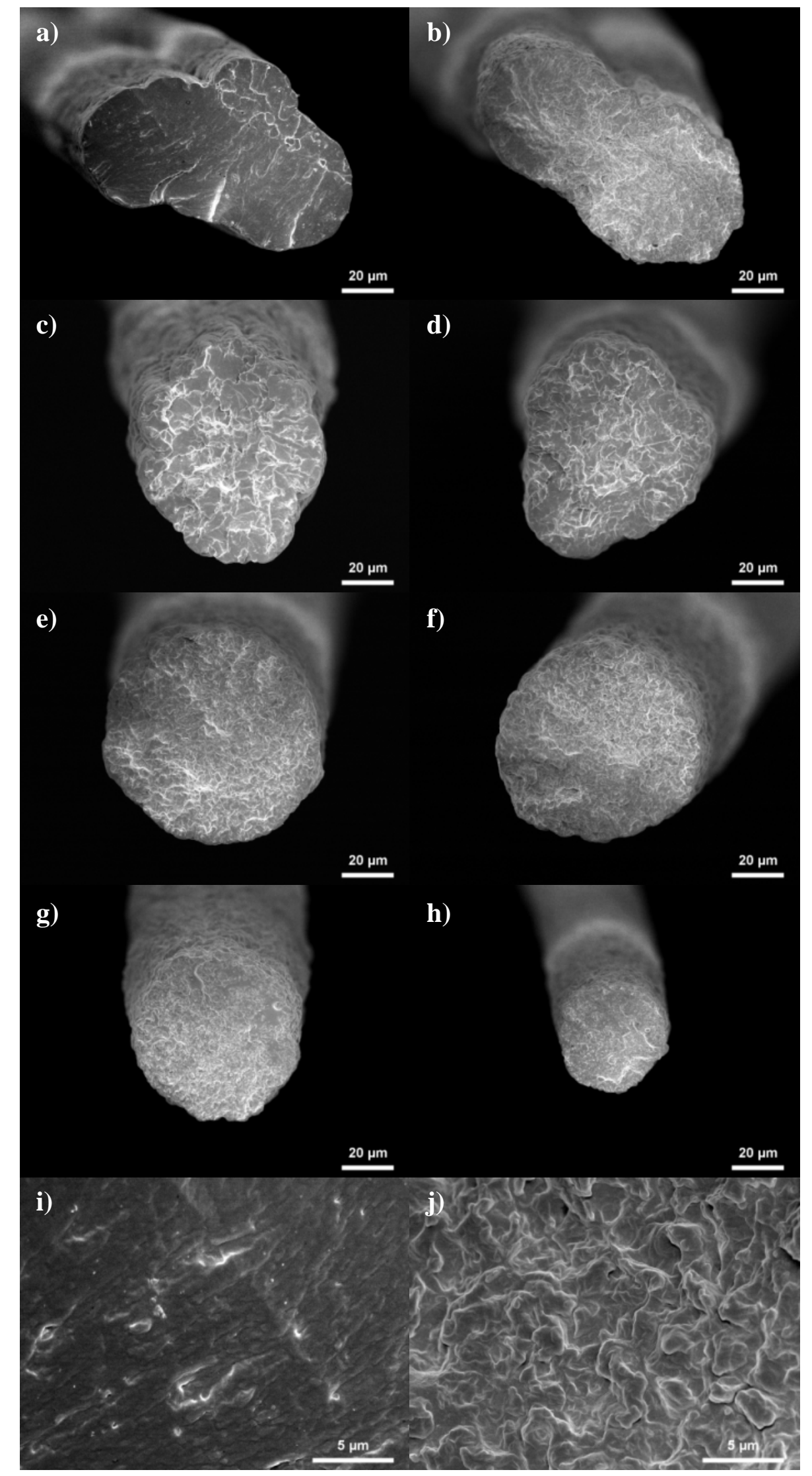

Figure 1. SEM micrographs of fibres produced from spinning formulations in DMSO into a coagulation bath of isopropanol/water $(80 / 20 \mathrm{v} / \mathrm{v})$ : a) pure PU fibre (No. 1), b-h) PU/PEDOT:PSS fibres with PEDOT:PSS loadings of b) 2.9 wt. \% (No. 3), c) 4.8 wt. \% (No. 
4), d) 9.1 wt. \% (No. 5), e) 13.0 wt. \% (No. 6), f) 16.7 wt. \% (No. 7), g) 20.0 wt. \% (No. 8), h) 25.0 wt. \% (No. 9), i) higher magnification of (a), and j) higher magnification of (e) 


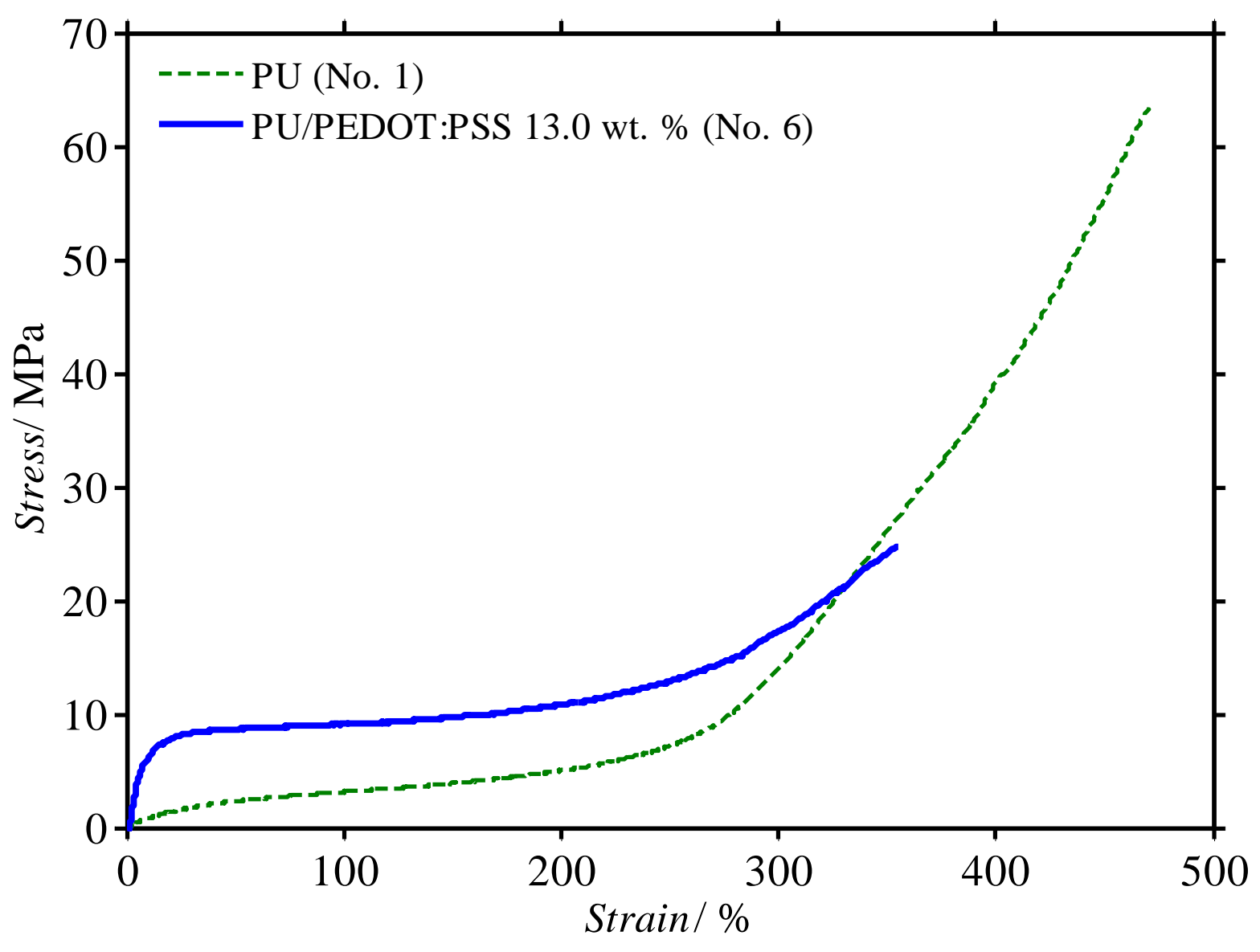

Figure 2. Stress-strain curves for tensile tests on PU fiber (No. 1) and PU/PEDOT:PSS fiber with 13.0 wt. \% PEDOT:PSS (No. 6) 


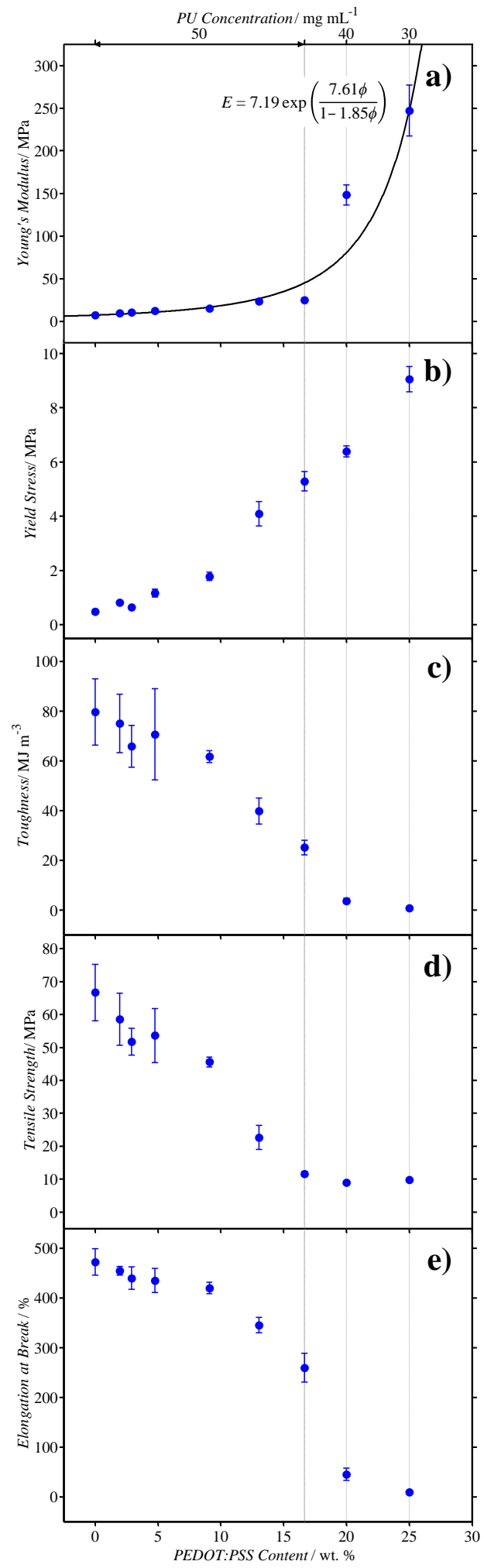

Figure 3. Mechanical properties of PU/PEDOT:PSS fibers at different PEDOT:PSS content: a) Young's modulus (the curve on experimental data represents the best Mooney's fit), b) yield stress, c) toughness, d) tensile strength, and e) elongation at break 

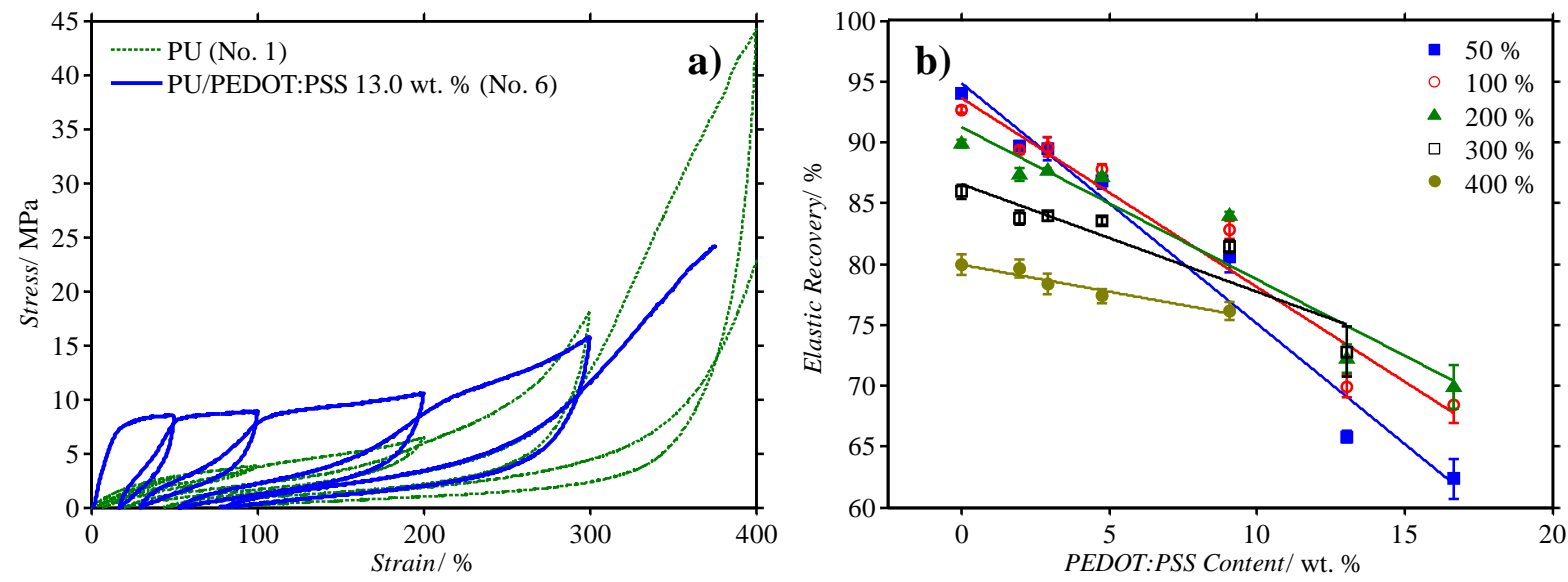

Figure 4. a) Stress strain curves for elastic recovery tests on PU fiber (No. 1) and PU/PEDOT:PSS fiber with 13.0 wt. \% PEDOT:PSS (No. 6), b) elastic recovery of PU/PEDOT:PSS fibers as a function of PEDOT:PSS content

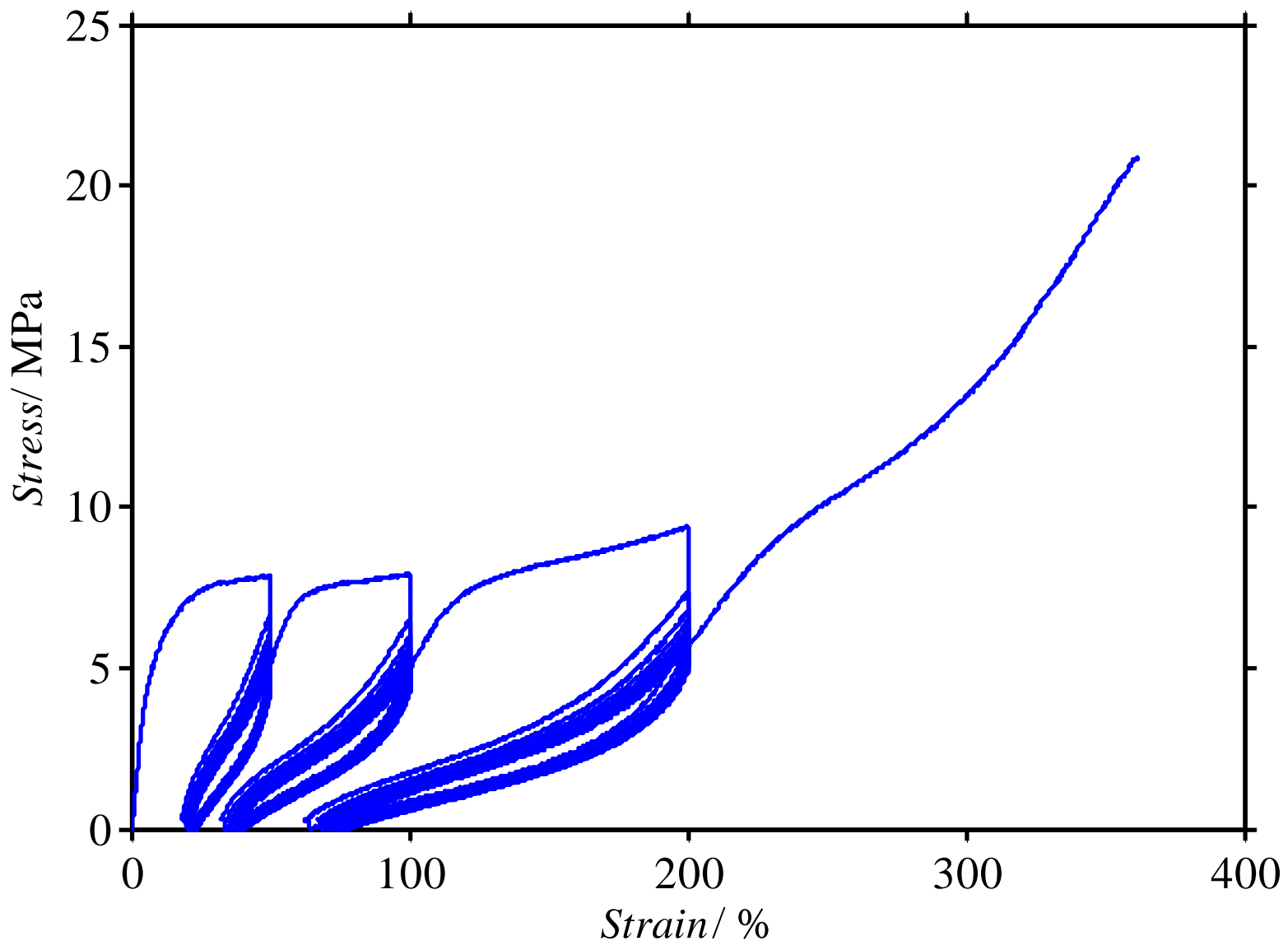

Figure 5. Cyclic test for PU/PEDOT:PSS fiber with 13.0 wt. \% PEDOT:PSS (No. 6) 


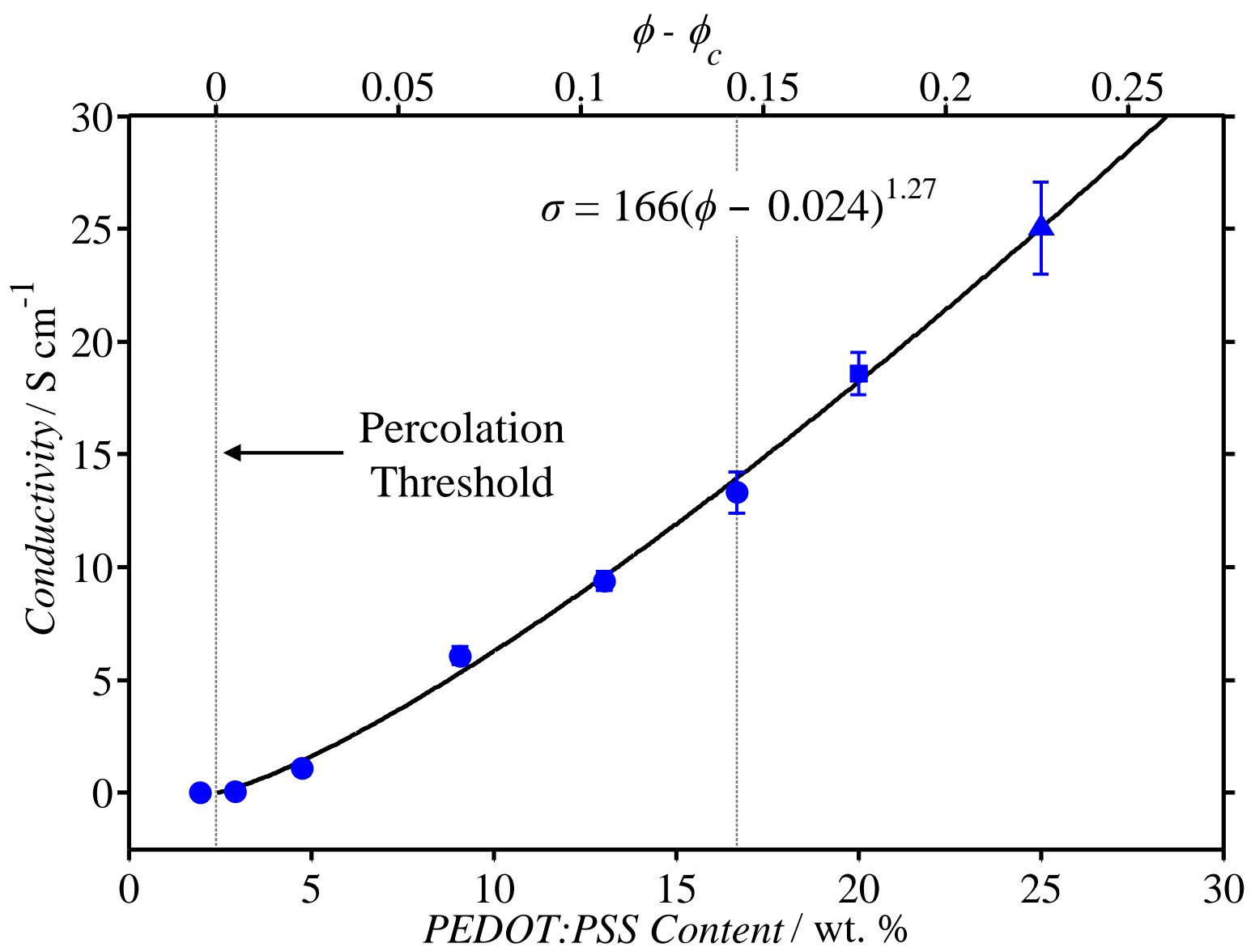

Figure 6. Conductivity of PU/PEDOT:PSS fibers at different PEDOT:PSS contents (• PU 50 $\mathrm{mg} \mathrm{mL}^{-1}$, $\boldsymbol{\nabla U} 40 \mathrm{mg} \mathrm{mL}^{-1}$, and $\boldsymbol{\Delta}$ PU $30 \mathrm{mg} \mathrm{mL}^{-1}$ ). The curve indicates the best fit obtained using percolation theory. 


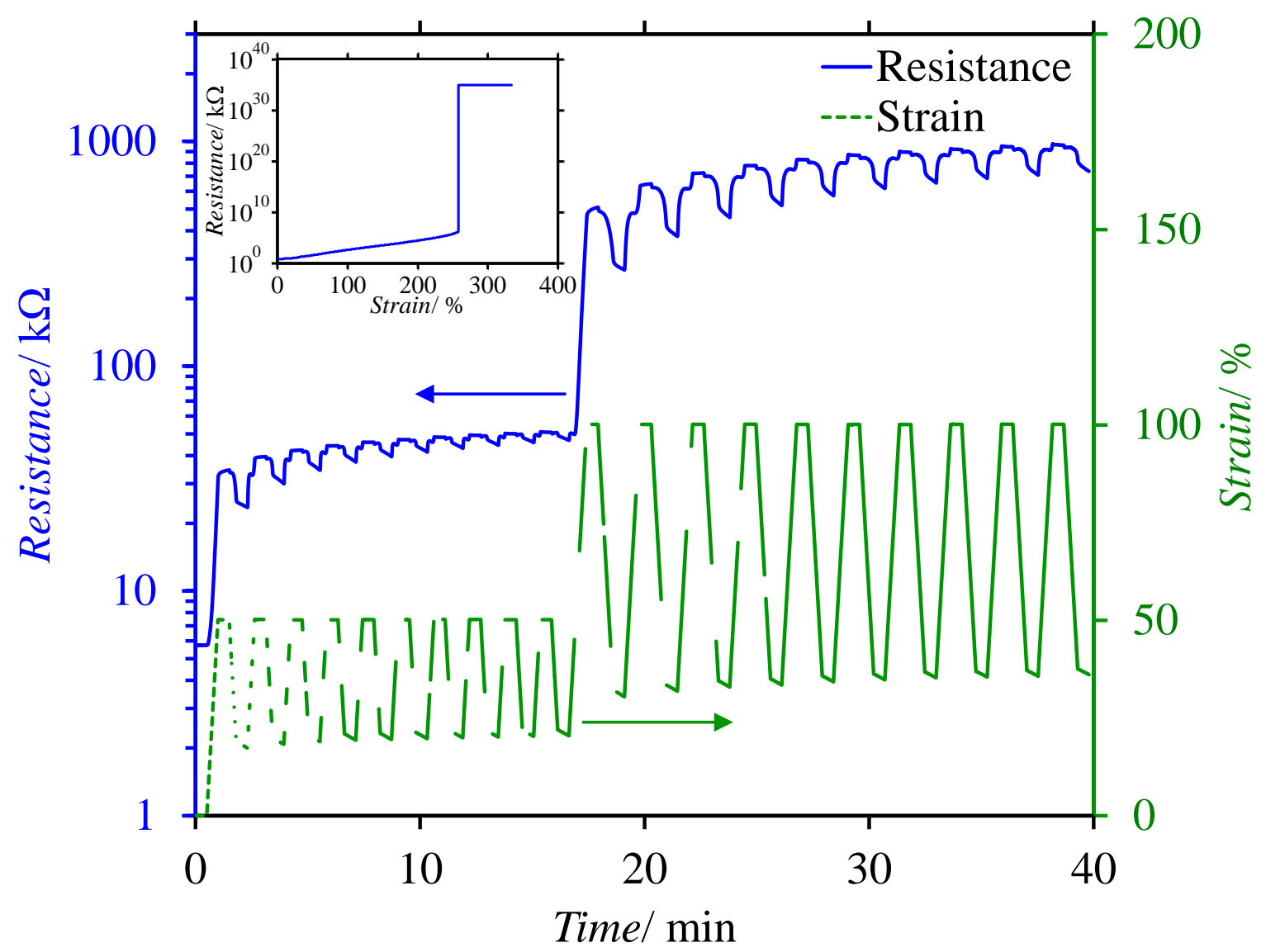

Figure 7. Resistance change by strain for PU/PEDOT:PSS fiber with 13.0 wt. \% PEDOT:PSS (No.6) in a cyclic electromechanical test at $50 \%$ and $100 \%$ strains for 10 cycles (inset shows the electromechanical tensile test for the same fiber) 


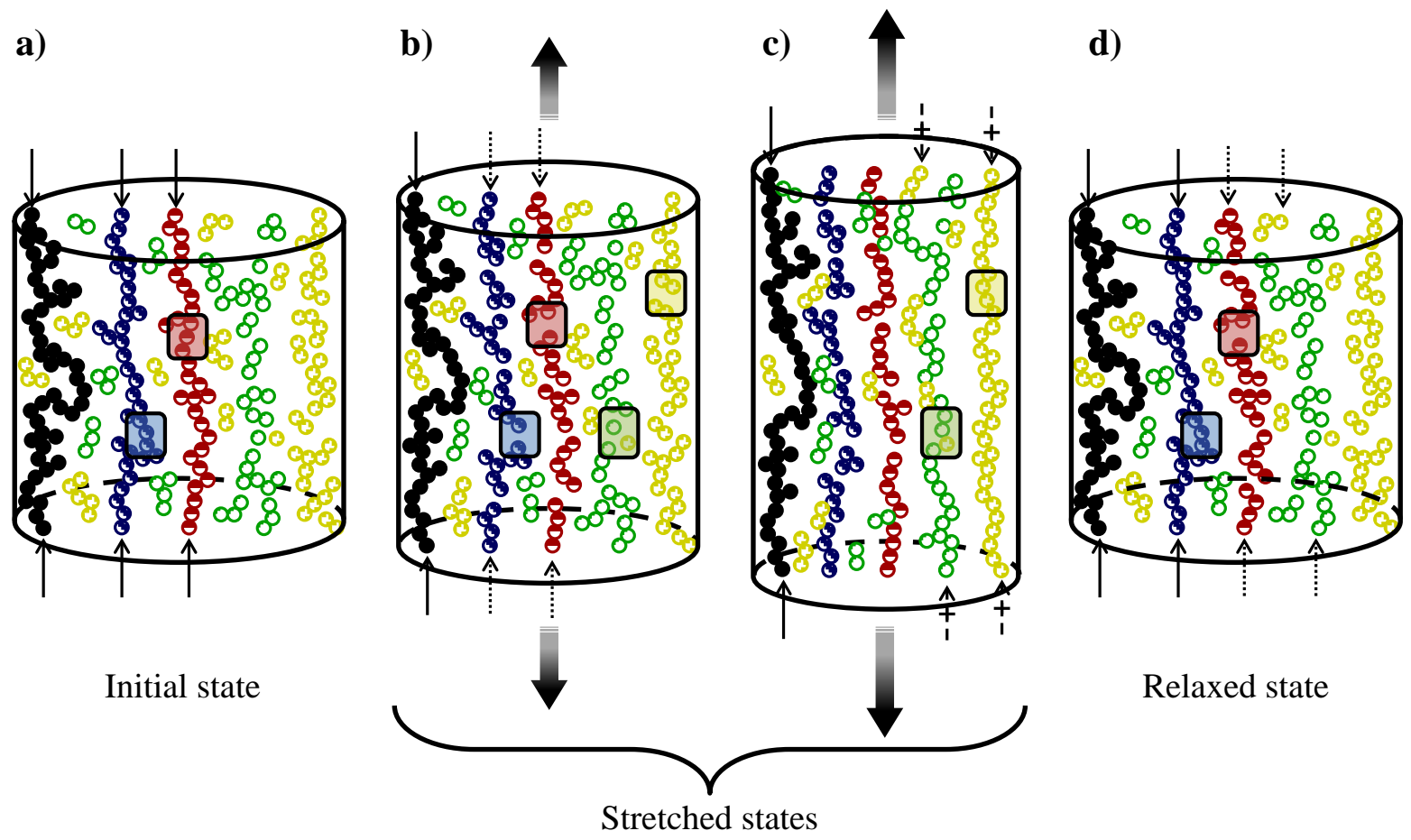

Figure 8. Schematic illustration of structural re-arrangement of PEDOT:PSS filler particle networks inside the PU host in their a) initial state, b,c) stretched states, and d) relaxed state. Different particle networks are shown in the figure $(\bullet$ Unbreakable networks, $\bullet$ Reversible networks, ○ Irreversible networks, ○ Isolated networks, and ๑ Debonded networks). $\uparrow \downarrow$ shows an existing conductive path, a newly established conductive path. 


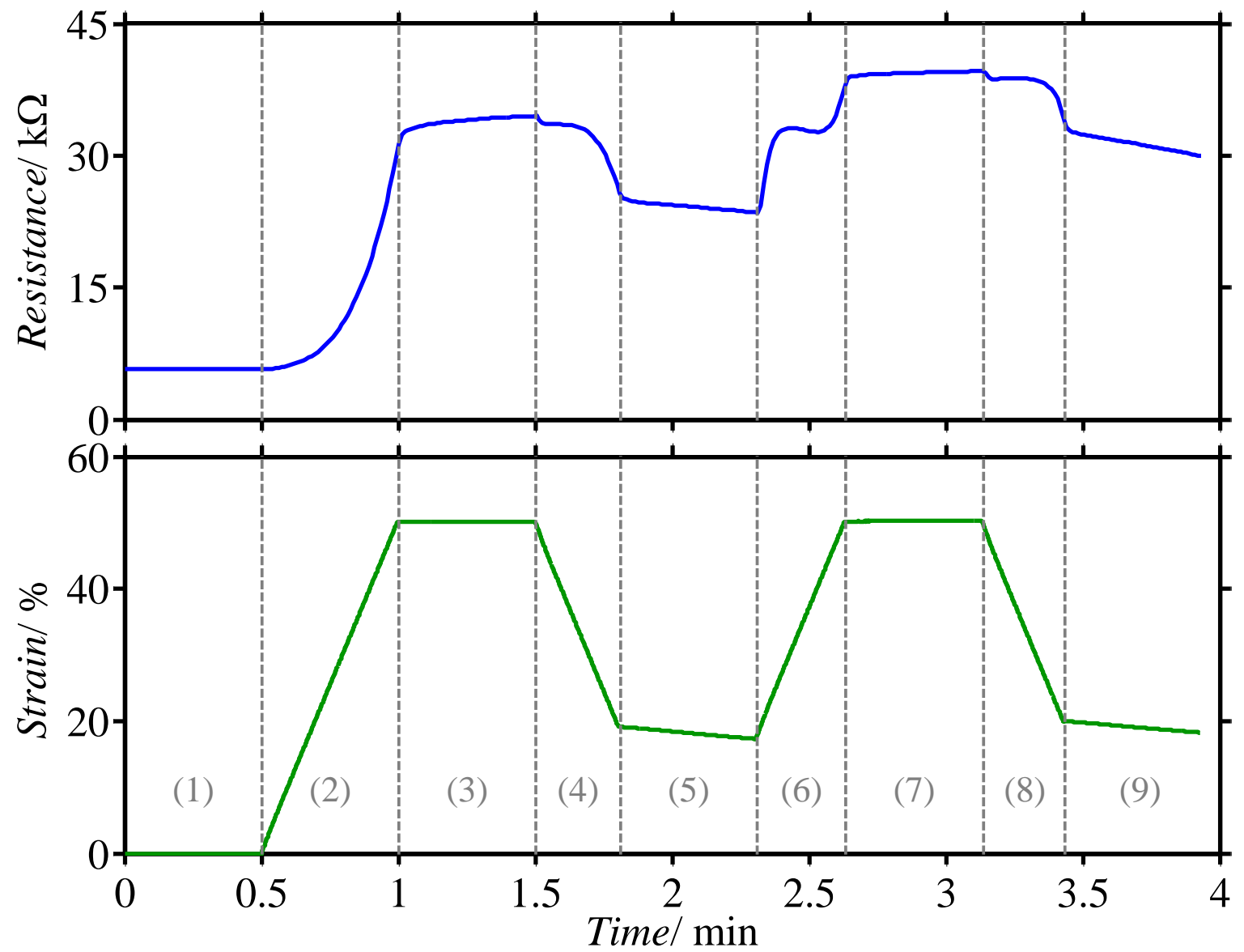

Figure 9. Resistance change by strain for PU/PEDOT:PSS fiber with 13.0 wt. \% PEDOT:PSS (No.6) in a cyclic electromechanical test under application of $50 \%$ for the first two cycles. Numbers show the stretching zones. 


\section{Table of Contents Entry:}

Conducting elastomeric fibers based on composite of polyurethane (PU) and PEDOT:PSS produced by wet-spinning method, have high electrical conductivity and stretchability. These fibers can sense large strains by changes in resistance. PU/PEDOT:PSS fiber is optimized to achieve the best strain sensing. PU/PEDOT:PSS fibers can be produced in large scale and integrated into conventional textile by weaving or knitting.

Keywords: Fibers, Sensors, Composite Materials, Conducting Polymers, Polymeric Materials M.Z. Seyedin, Joselito M. Razal*, Peter C. Innis, and Gordon G. Wallace*

Strain responsive polyurethane/PEDOT:PSS conducting elastomeric composite fibers

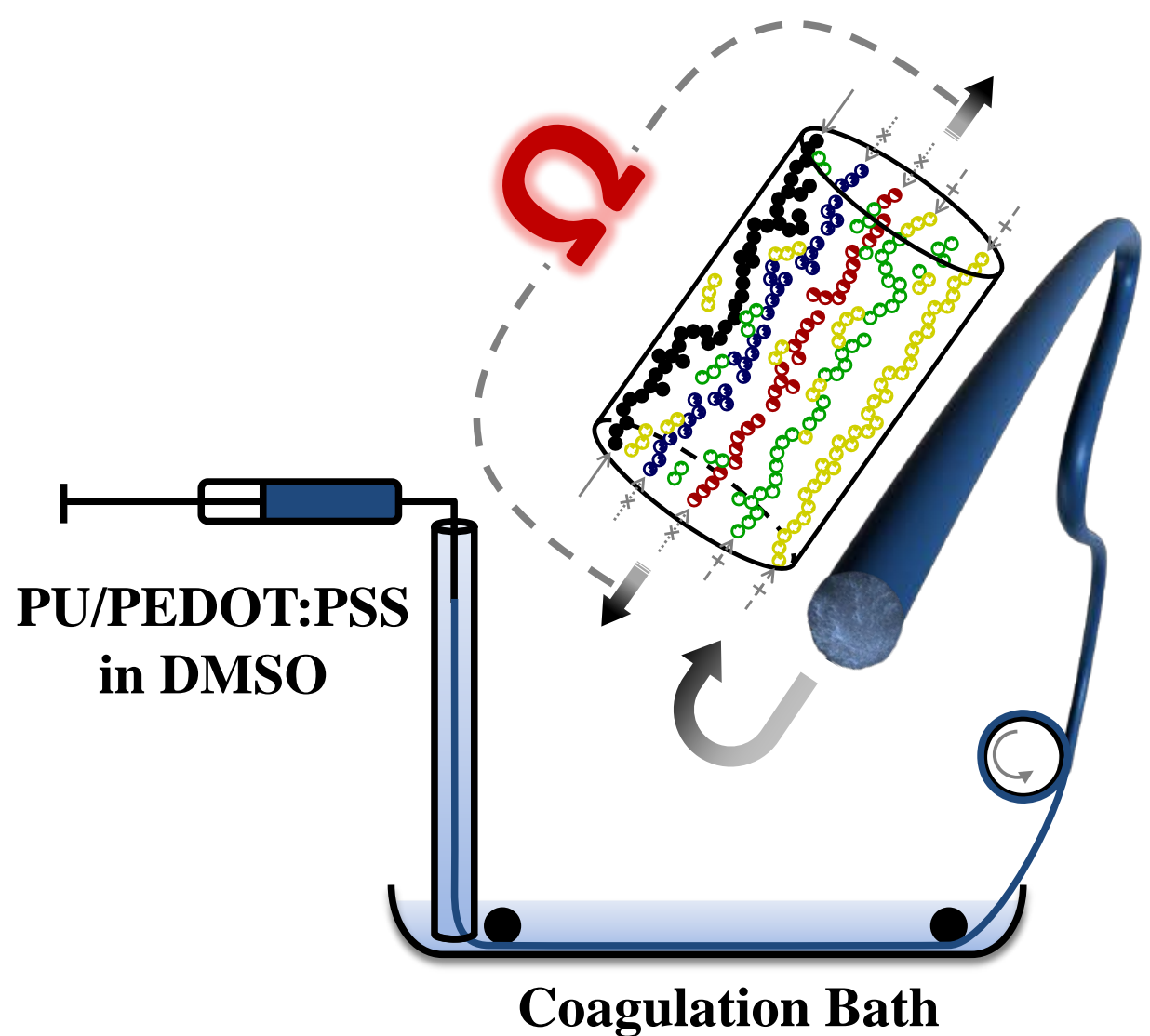


Supporting Information for

\section{Strain-responsive polyurethane/PEDOT:PSS elastomeric composite fibers with high electrical conductivity}

By Mohammad Ziabari Seyedin, Joselito M. Razal*, Peter C. Innis, and Gordon G. Wallace*

Intelligent Polymer Research Institute

ARC Centre of Excellence for Electromaterials Science

AIIM Facility, Innovation Campus

University of Wollongong

Wollongong NSW 2522 (Australia)

E-mail: jrazal@uow.edu.au, gwallace@uow.edu.au

\section{Particle size of PEDOT:PSS dispersions}
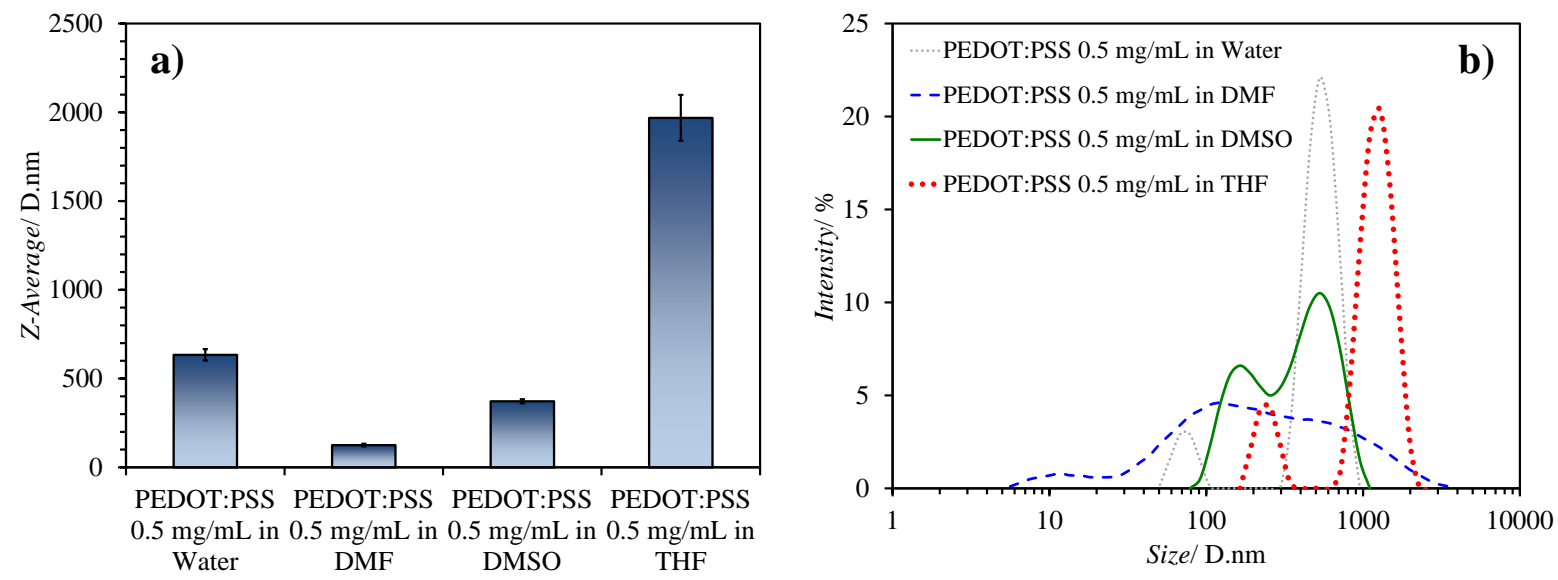

Figure S1. a) Z-Average and b) particle size distribution of PEDOT:PSS particles in different solvents at concentration of $0.5 \mathrm{mg} \mathrm{mL}^{-1}$ 

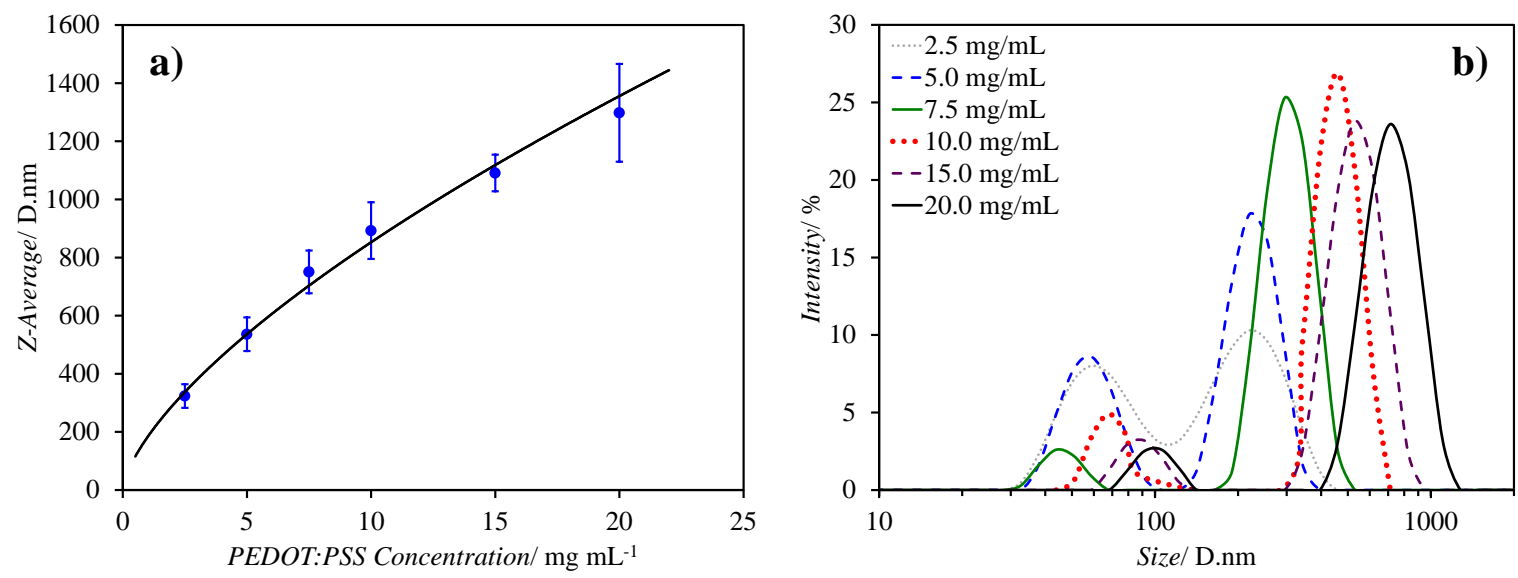

Figure S2. a) Z-Average (the line on data represents the best fit using a power-law relationship) and b) particle size distribution of PEDOT:PSS particles in DMSO at different concentrations

\section{Conductivity of PEDOT:PSS drop-cast films}

Drop-cast films were prepared from PEDOT:PSS dispersions $\left(15 \mathrm{mg} \mathrm{mL}^{-1}\right)$ in different solvents on plasma-cleaned (Harrick Plasmaflo PDC-FMG) glass slides. $300 \mu$ l of each dispersion was transferred on the surface of the glass slide and was left to dry (water: at room temperature for $24 \mathrm{~h}$, DMSO: at $100{ }^{\circ} \mathrm{C}$ on a hot plate for $5 \mathrm{~min}, \mathrm{DMF}$ : at $75^{\circ} \mathrm{C}$ on a hot plate for $5 \mathrm{~min}$ and THF: at room temperature for $30 \mathrm{~min}$ ). Thickness and resistivity of PEDOT:PSS films were measured by an optical profiler (Veeco Wyko NT9100) and fourpoint probe resistivity measurement system (Jandel RM2) with a linear probe head, which were then used to calculate conductivity of the films from a minimum of 10 points. 


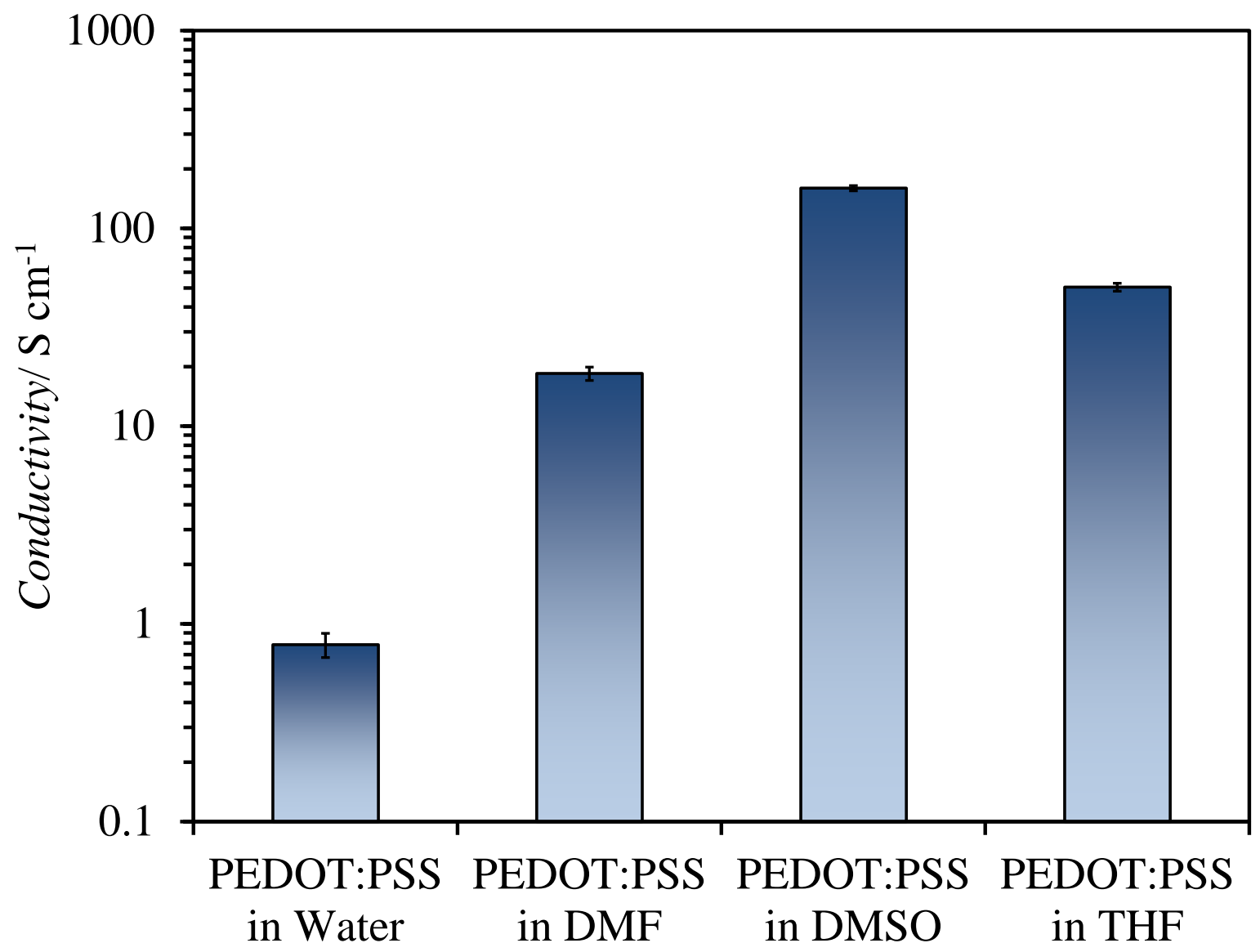

Figure S3. Conductivity of pristine PEDOT:PSS drop-cast films obtained from different dispersions

\section{Continuous PU/PEDOT:PSS fiber fabrication}

Fabrication of PU/PEDOT:PSS fibers were continuous and uniform fibers were obtained at all spinnable formulations $(0-25.0$ wt. \%). Figure S4 illustrates the PU/PEDOT:PSS fiber at 13.0 wt. \% PEDOT:PSS as-spun on a winder produced by the wet-spinning approach. Continuous fabrication of PU/PEDOT:PSS fibers enables the integration into textiles by conventional weaving, knitting, or braiding techniques. 


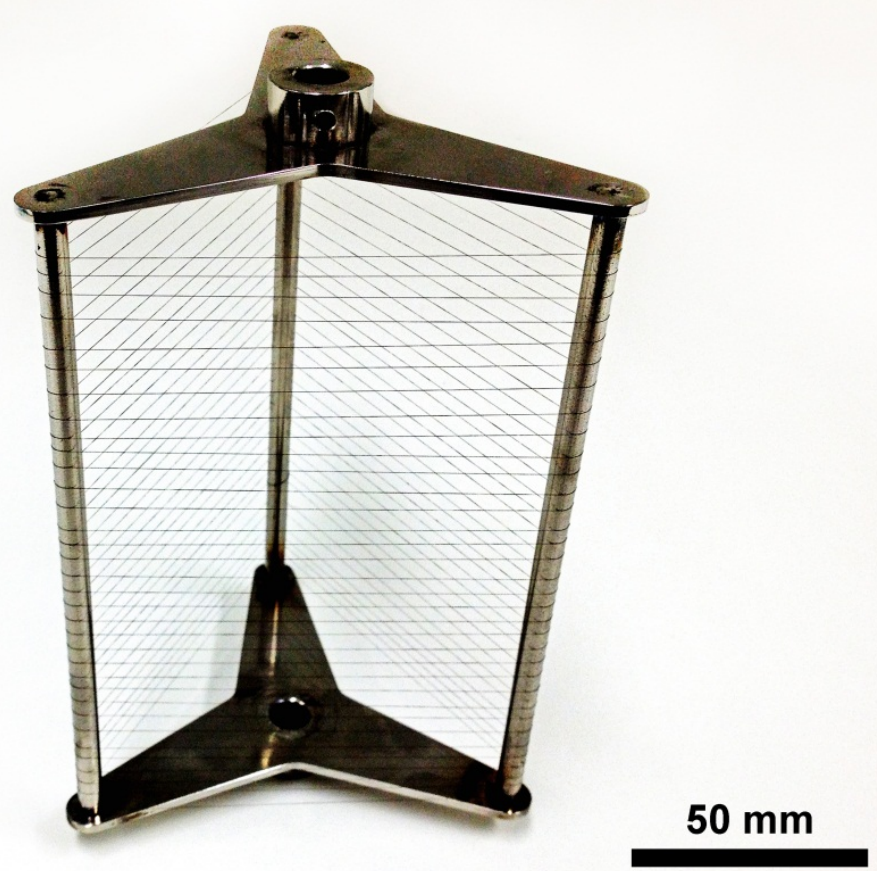

Figure S4. Wet-spun PU/PEDOT:PSS fiber with 13.0 wt. \% PEDOT:PSS (No.6)

The average fiber diameters obtained from solutions with PU concentration of $50 \mathrm{mg} \mathrm{mL} \mathrm{m}^{-1}$ (samples No. 1 - 7) remained constant at 80 - $85 \mu$ m even after loading with PEDOT:PSS (Table S1). However, a more uniform diameter along the length of the fibers were observed with higher PEDOT:PSS loadings. This was presumably a result of a slower coagulation rate as well as higher spinnability due to an increased viscosity of the spinning solution (see Figure 3). Fibers diameters decreased for samples No. 8 and 9 where the lower concentrations of PU were used as a result of decreased viscosity. 


\section{The effect of PEDOT:PSS particle size on mechanical properties of PU/PEDOT:PSS}

fibers

The effect of PEDOT:PSS particle sizes on mechanical properties of PU/PEDOT:PSS fibers was studied by preparing two spinning solutions with same concentrations of components made from PEDOT:PSS dispersions with different concentrations, namely 15 and $20 \mathrm{mg} \mathrm{mL}{ }^{-}$ ${ }^{1}$, and therefore different particle sizes (Figure S2). PU/PEDOT:PSS fiber obtained from a spinning mixture of $15 \mathrm{mg} \mathrm{mL}^{-1}$ dispersion of PEDOT:PSS (lower particle size) and $50 \mathrm{mg}$ $\mathrm{mL}^{-1} \mathrm{PU}$ (sample No. 7) had a Young’s modulus of $24.88 \pm 2.49 \mathrm{MPa}$, elongation at break of $259 \pm 29 \%$, tensile strength of $11.59 \pm 0.74 \mathrm{MPa}$, and toughness of $25.1 \pm 2.9 \mathrm{MJ} \mathrm{m}^{-3}$. This fiber showed superior mechanical properties to its counterpart obtained from higher PEDOT:PSS dispersion concentration of $20 \mathrm{mg} \mathrm{mL}^{-1}$ (higher particle size, mixed to result in the same concentration of PU) with a measured Young's modulus of $24.8 \pm 1.9 \mathrm{MPa}$, elongation at break of $95 \pm 23 \%$, tensile strength of $9.41 \pm 0.46 \mathrm{MPa}$, and toughness of $8.0 \pm$ 1.9 $\mathrm{MJ} \mathrm{m}^{-3}$. As both fibers had a very similar Young's modulus, it can be deduced that they have the same degree of reinforcement, suggesting an equally uniform distribution of PEDOT:PSS and efficient stress transfer at low strains for both systems. This also implies that the loading of PEDOT:PSS (hydrodynamic effect) was the major factor influencing Young's modulus of the fibers. However, elongation at break, toughness, and tensile strength were significantly affected. This could be due to thwarted strain induced crystallization as a result of adding $20 \mathrm{mg} \mathrm{mL}^{-1}$ PEDOT:PSS dispersion to PU. Relating these observations to particle sizes of the dispersions, lower mechanical properties of fibers obtained from the composite fiber prepared from PEDOT:PSS dispersion of $20 \mathrm{mg} \mathrm{mL}^{-1}$ compared to that of $15 \mathrm{mg} \mathrm{mL}^{-1}$, could be attributed to larger particle sizes in the former. Therefore, it could be inferred that spinning solutions prepared from higher concentrations of PEDOT:PSS in the dispersion, which contain larger particles, will result in lower mechanical properties in the final fibers. 
This result suggests that quality of PEDOT:PSS dispersion has a profound impact on mechanical properties of PU/ PEDOT:PSS fibers.

\section{Elastic recovery of PU/PEDOT:PSS fibers}

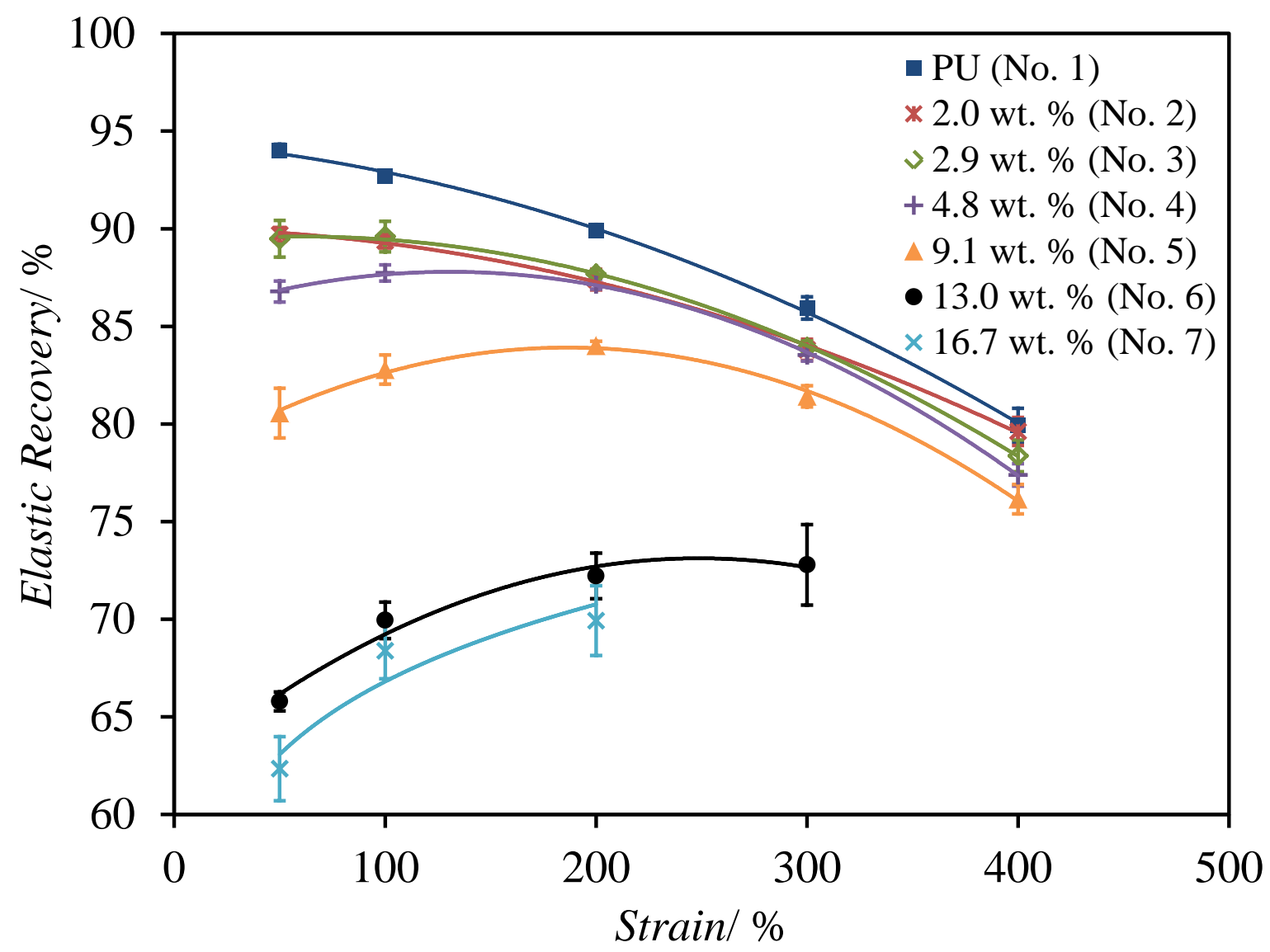

Figure S5. Elastic recovery of PU and PU/PEDOT:PSS fibers as a function of applied strains 


\section{Viscosity of PU/PEDOT:PSS spinning formulations}

Viscosity of spinning formulations (Figure 3) was measured by a rheometer (TA Instruments AR-G2) at $25^{\circ} \mathrm{C}$ using a cone and plate geometry with a cone having an angle of $2^{\circ}$, diameter of $40 \mathrm{~mm}$, and truncation of $55 \mu \mathrm{m}$.

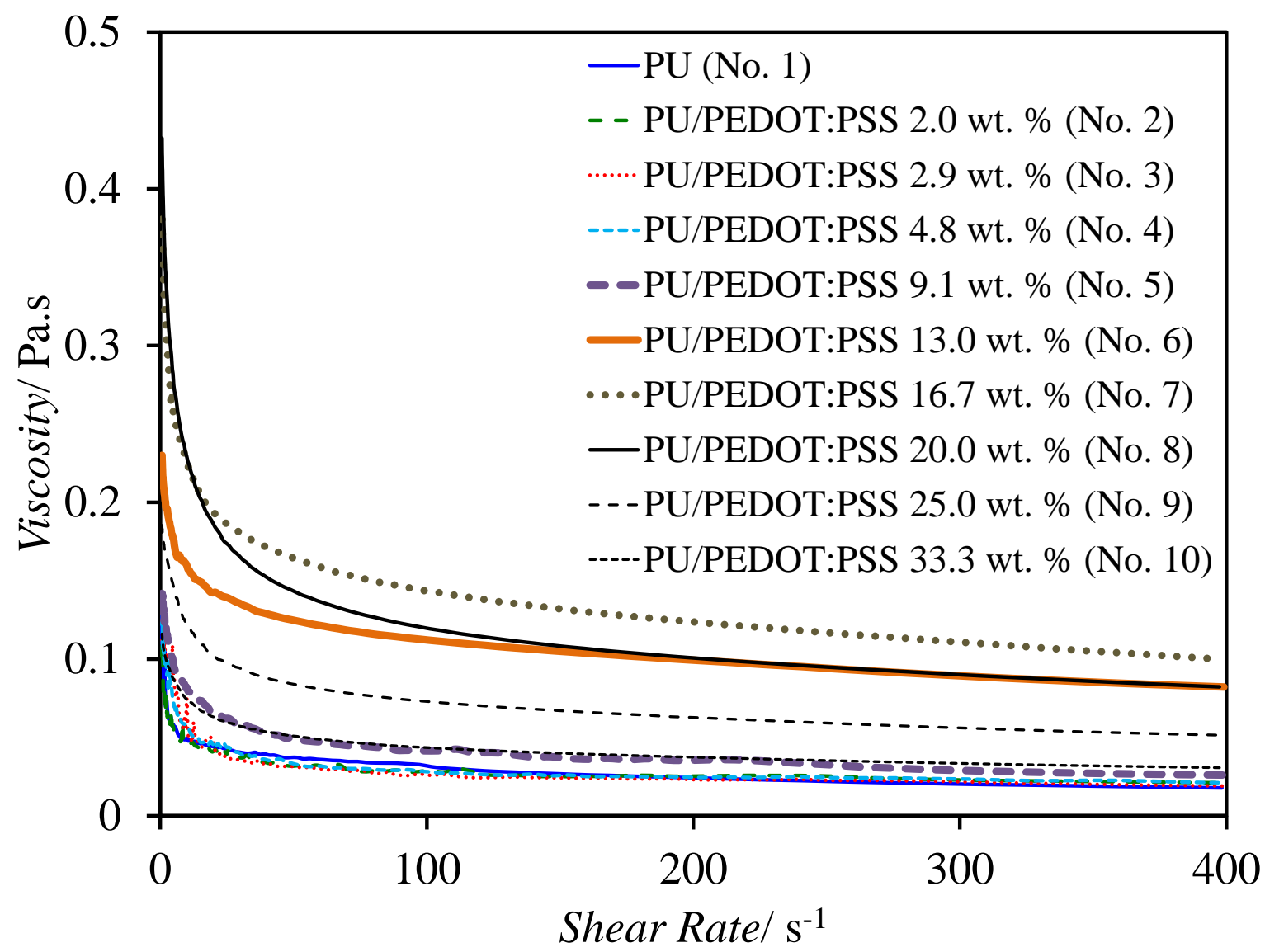

Figure S6. Viscosity vs. shear rate for PU/PEDOT:PSS spinning solutions in DMSO at different concentrations 


\section{Electrical and mechanical properties of PU/PEDOT:PSS fibers from different spinning formulations}

Electrical and mechanical properties of our optimized PU/PEDOT:PSS fibers are listed in Table S1 at different loadings of PEDOT:PSS.

Table S1. Electrical and mechanical properties of PU/PEDOT:PSS fibers wet-spun from DMSO with coagulation bath of isopropanol/water (80/20 v/v)

Jo. PU

PEDOT: PEDOT: Fibers Electrical Young's Yield

PSS PSS Diameter Conductivity Modulus Stress at Break Strength at $50 \%$ at $100 \%$ at $200 \%$ at $300 \%$ at $400 \%$

Toughness

Elongation Tensile Elastic Recovery $\left[\mathrm{mg} \mathrm{mL}^{-}\left[\mathrm{mg} \mathrm{mL}^{-}\right.\right.$

1] $\quad 1$

[wt. \% $] \quad[\mu \mathrm{m}] \quad\left[\mathrm{S} \mathrm{cm}^{-1}\right] \quad[\mathrm{MPa}] \quad[\mathrm{MPa}] \quad\left[\mathrm{MJ} \mathrm{m}^{-3}\right] \quad[\%$

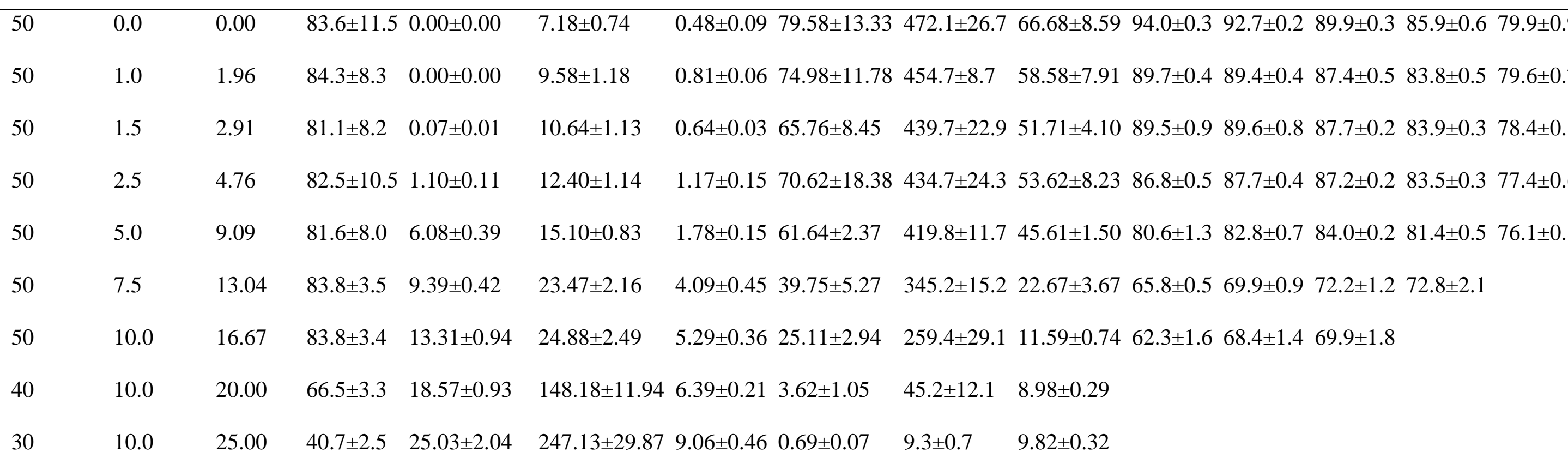


We also produced PU/PEDOT:PSS fibers from the DMF and THF dispersions. PU/PEDOT:PSS fiber formation was also possible in the presence of polyethylene glycol (PEG) as the secondary solvent in the spinning formulations. PU/PEDOT:PSS fibers wet-spun from the DMF and THF dispersions were inferior to their DMSO counterparts. Table S2 lists the conditions investigated and conductivities achieved.

Table S2. PU/PEDOT:PSS fiber formation from DMF and THF at different conditions

\begin{tabular}{|c|c|c|c|c|c|c|c|}
\hline 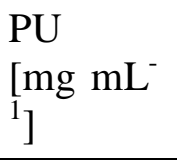 & $\begin{array}{l}\text { PEDOT:PSS } \\
{\left[\mathrm{mg} \mathrm{mL}^{-1}\right]}\end{array}$ & $\begin{array}{l}\text { PEDOT:PSS } \\
\text { [wt. \%] }\end{array}$ & Solvent & $\begin{array}{l}\text { PEG } \\
\text { [vol. } \\
\% \text { ] }\end{array}$ & $\begin{array}{l}\text { Coagulation } \\
\text { Bath }\end{array}$ & $\begin{array}{l}\text { Flow } \\
\text { Rate } \\
{\left[\mathrm{mL} \mathrm{h}^{-1}\right]}\end{array}$ & $\begin{array}{l}\text { Conductivity } \\
{\left[\mathrm{S} \mathrm{cm}^{-1}\right]}\end{array}$ \\
\hline 50 & 5 & 9.1 & DMF & - & $\mathrm{EtOH}(\mathrm{H})$ & 0.5 & $0.06 \pm 0.02$ \\
\hline 50 & 5 & 9.1 & DMF & - & $\mathrm{EtOH}(\mathrm{V})$ & $0.1-10$ & No fibre \\
\hline 50 & 10 & 16.7 & $\mathrm{DMF}$ & - & Water $(\mathrm{H})$ & $0.1-10$ & No fibre \\
\hline 50 & 10 & 16.7 & DMF & - & $\mathrm{EtOH}(\mathrm{H})$ & 0.5 & $1.41 \pm 0.37$ \\
\hline 50 & 10 & 16.7 & $\mathrm{DMF}$ & - & IPA (V) & 5 & $0.55 \pm 0.10$ \\
\hline 50 & 10 & 16.7 & DMF & 10 & $\mathrm{EtOH}(\mathrm{H})$ & 0.5 & $1.75 \pm 0.53$ \\
\hline 50 & 10 & 16.7 & DMF & 10 & IPA (V) & 5 & $2.29 \pm 0.10$ \\
\hline 50 & 15 & 23.1 & $\mathrm{DMF}$ & - & $\mathrm{EtOH}(\mathrm{H})$ & 0.5 & $3.64 \pm 1.08$ \\
\hline 50 & 15 & 23.1 & $\mathrm{DMF}$ & - & IPA (V) & 5 & $3.12 \pm 0.59$ \\
\hline 30 & 10 & 25.0 & DMF & - & $\mathrm{EtOH}(\mathrm{H})$ & $0.1-10$ & No Fibre \\
\hline 30 & 10 & 25.0 & $\mathrm{DMF}$ & - & IPA (V) & 5 & $7.45 \pm 0.72$ \\
\hline 20 & 10 & 33.3 & $\mathrm{DMF}$ & - & EtOH $(\mathrm{H})$ & $0.1-10$ & No fibre \\
\hline 20 & 10 & 33.3 & DMF & - & IPA (V) & $0.1-10$ & No fibre \\
\hline 50 & 10 & 16.7 & $\mathrm{THF}$ & - & $\mathrm{EtOH}(\mathrm{H})$ & 0.5 & $5.39 \pm 0.71$ \\
\hline 50 & 10 & 16.7 & THF & - & IPA (V) & 5 & $3.61 \pm 0.21$ \\
\hline
\end{tabular}

(H): Horizontal wet-spinning setup

(V): Horizontal wet-spinning setup

EtOH: Ethanol

IPA: Isopropanol 